\title{
Modified method of simplest equation for obtaining exact analytical solutions of nonlinear partial differential equations: Further development of methodology with two applications
}

\author{
Nikolay K. Vitanov ${ }^{1,2, *}$ Zlatinka I. Dimitrova ${ }^{3}$, Kaloyan N. Vitanov ${ }^{1}$ \\ ${ }^{1}$ Institute of Mechanics, Bulgarian Academy of Sciences, \\ Acad. G. Bonchev Str., Bl. 4, 1113 Sofia, Bulgaria \\ 2 Max-Plank Institute for the Physics of Complex Systems, \\ Nöthnitzer Str. 38, 01187 Dresden, Germany \\ 3 "G. Nadjakov" Institute of Solid State Physics, Tzarigradsko \\ Chaussee Blvd. 72, 1784 Sofia, Bulgaria
}

\begin{abstract}
We discuss the application of a variant of the method of simplest equation for obtaining exact traveling wave solutions of a class of nonlinear partial differential equations containing polynomial nonlinearities. As simplest equation we use differential equation for a special function that contains as particular cases trigonometric and hyperbolic functions as well as the elliptic function of Weierstrass and Jacobi. We show that for this case the studied class of nonlinear partial differential equations can be reduced to a system of two equations containing polynomials of the unknown functions. This system may be further reduced to a system of nonlinear algebraic equations for the parameters of the solved equation and parameters of the solution. Any nontrivial solution of the last system leads to a traveling wave solution of the solved nonlinear partial differential equation. The methodology is illustrated by obtaining solitary wave solutions for the generalized Korteweg-deVries equation and by obtaining solutions of the higher order Korteweg-deVries equation.
\end{abstract}

*corresponding author, e-mail: vitanov@imbm.bas.bg 


\section{Introduction}

Nonlinearity is an essential feature of many systems in Nature and society [1] - [13]

Traveling wave solutions of nonlinear partial differential equations are studied much in the last decades [14]-[21] as they occur in many natural systems [22]-25] and because of existence of various methods for obtaining such solutions [26] - 30]. Below we shall consider the method of simplest equation for obtaining exact analytical solutions of nonlinear partial differential equations 31-34 and especially its version called modified method of simplest equation [35] - [37]. Method of simplest equation is based on a procedure analogous to the first step of the test for the Painleve property [34], [38]. In the version of the method called modified method of the simplest equation [39], [40] this procedure is substituted by the concept for the balance equation. Modified method of simplest equation has its roots back in the history (for an example see [41]-444]). Method of simplest equation has been successfully applied for obtaining exact traveling wave solutions of numerous nonlinear PDEs such as versions of generalized Kuramoto - Sivashinsky equation, reaction - diffusion equation, reaction - telegraph equation, generalized Swift - Hohenberg equation and generalized Rayleigh equation, generalized Fisher equation, generalized Huxley equation, generalized Degasperis - Procesi equation and b-equation, extended Korteweg-de Vries equation, etc. [45] - [51].

A short summary of the method of simplest equation is as follows. First of all by means of an appropriate ansatz (for an example the traveling-wave ansatz) the solved of nonlinear partial differential equation is reduced to a nonlinear ordinary differential equation

$$
P\left(u, u_{\xi}, u_{\xi \xi}, \ldots\right)=0
$$

Then the finite-series solution

$$
u(\xi)=\sum_{\mu=-\nu}^{\nu_{1}} p_{\mu}[g(\xi)]^{\mu}
$$

is substituted in (1.1). $p_{\mu}$ are coefficients and $g(\xi)$ is solution of simpler ordinary differential equation called simplest equation. Let the result of this substitution be a polynomial of $g(\xi)$. Eq. (1.2) is a solution of Eq.(1.1) if all coefficients of the obtained polynomial of $g(\xi)$ are equal to 0 . This condition leads to a system of nonlinear algebraic equations. Each solution of the last system leads to a solution of the studied nonlinear partial differential equation. 
In this article we consider a large class of $(1+1)$-dimensional nonlinear partial differential equations that are constricted by polynomials of the unknown function and its derivatives. As simplest equation we shall use equation of the kind

$$
\left(\frac{d g}{d \xi}\right)^{2}=\sum_{i=0}^{m} a_{i} g^{i}
$$

The text below is organized as follows. In Sect. 2 we introduce the class of studied nonlinear partial differential equations and the used class of simplest equations and their solutions. Then we show that any of the nonlinear partial differential equations of the discussed class can be reduced to a system of two equations containing polynomials of the unknown function. These polynomials can be obtained on the basis of addition and multiplication of some basic polynomials connected to the derivatives of the solved nonlinear partial differential equation. In section 3 we calculate some of the most used basic polynomials. In Sect. 4 the methodology is illustrated by application for obtaining solitary wave solutions of

- generalized Korteweg-deVries equation;

- higher order Korteweg-deVries equation.

Several concluding remarks are summarized in Sect. 5 .

\section{Formulation of the method}

\subsection{Proof of the basic theorem}

Let us consider a nonlinear PDE with nonlinearities that are polynomials of the unknown function $h(x, t)$ and its derivatives. We search solution of the kind

$$
h(x, t)=h(\xi) ; \quad \xi=\mu x+\nu t
$$

where $\mu$ and $\nu$ are parameters. The basis of our search will be a solution $g(\xi)$ of a certain simplest equation. Hence

$$
h=f[g(\xi)]
$$

$h$ from Eq.(2.2) is a composite function. For the $n$-th derivative of $h$ we have the Faa di Bruno formula 52.

$$
h_{(n)}=\sum_{k=1}^{n} f_{(k)} \sum_{p(k, n)} n ! \prod_{i=1}^{n} \frac{g_{(i)}^{\lambda_{i}}}{\left(\lambda_{i} !\right)(i !)^{\lambda_{i}}}
$$

where 
- $h_{(n)}=\frac{d^{n} h}{d x^{n}}$

- $f_{(k)}=\frac{d^{k} f}{d g^{k}}$

- $g_{(i)}=\frac{d^{i} g}{d x^{i}}$

- $p(n, k)=\left\{\lambda_{1}, \lambda_{2}, \ldots, \lambda_{n}\right\}$ : set of numbers such that

$$
\sum_{i=1}^{n} \lambda_{i}=k ; \sum_{i=1}^{n} i \lambda_{i}=n .
$$

Further we shall concentrate on $f_{(k)}$ and $g_{(i)}$.

Let us now assume that $f$ is a polynomial of $g$. Then

$$
f=\sum_{r=0}^{q} b_{r} g^{r}
$$

Let us consider the derivative $f_{(k)}$. If $k>r$ this derivative is 0 . The derivative is non-zero if $k \leq r$. We shall use the function $\Theta_{r k}$ with the following definition

$$
\Theta_{r k}= \begin{cases}0 & r<k \\ 1 & r \geq k\end{cases}
$$

Then the derivative $f_{(k)}$ can be written as

$$
f_{(k)}=\sum_{r=0}^{q} \Theta_{r k} \frac{r !}{(r-k) !} b_{r} g^{r-k}
$$

The derivative $g_{(i)}$ is connected to the simplest equation. In general we can use the following simplest equation with polynomial nonlinearity

$$
g_{(k)}^{l}=\left(\frac{d^{k} g}{d \xi^{k}}\right)^{l}=\sum_{j=0}^{m} a_{j} g^{j}
$$

The solution of this equation defines certain special function $V_{a_{0}, a_{1}, \ldots, a_{m}}(\xi ; k, l, m)$ where

- $k$ : order of derivative of $g$;

- $l$ : degree of derivative in the defining ODE;

- $m$ : highest degree of the polynomial of $g$ in the defining ODE. 
This special function has very interesting properties as its particular cases are the trigonometric, hyperbolic, elliptic functions of Jacobi, etc. Below we shall use the function $V_{a_{0}, a_{1}, \ldots, a_{m}}(\xi ; 1,2, m)$ which is solution of the simplest equation

$$
g_{(1)}^{2}=\left(\frac{d g}{d \xi}\right)^{2}=\sum_{j=0}^{m} a_{j} g^{j}
$$

We let $m$ undetermined for now. $g_{(i)} ?$

If $g_{(1)}^{2}$ is given by Eq.(2.9) then what is the relationship for the derivative

Lemma. If $g_{(1)}^{2}$ is given by Eq. (2.9) then the following relationship holds for the derivative $g_{(i)}$ :

$$
g_{(i)}^{\lambda_{i}}=\left[A_{i}(g)\right]^{\lambda_{i}} O_{i, \lambda_{i}}\left(g, g_{(1)}\right) \Omega_{\lambda_{i}}\left(g_{(1)}\right)
$$

where

- $\lambda_{i}$ is a non-negative integer;

- $A_{i}(g)$ : polynomial of $g$;

$$
O_{i, \lambda_{i}}\left(g, g_{(1)}\right)= \begin{cases}1 & i-\text { even, } \lambda_{i}-\text { even } \\ \frac{1}{g_{(1)}} & i-\text { even, } \lambda_{i}-\text { odd } \\ \left(\sum_{j=0}^{m} a_{j} g^{j}\right)^{\left[\lambda_{i} / 2\right]} & i-\text { odd }, \lambda_{i}-\text { even } \\ \left(\sum_{j=0}^{m} a_{j} g^{j}\right)^{\left[\lambda_{i} / 2\right]} & i-\text { odd }, \lambda_{i}-\text { odd }\end{cases}
$$

where $\left[\lambda_{i} / 2\right]$ denotes the integer part of $\lambda_{i} / 2$;

$$
\Omega_{i}\left(g_{(1)}\right)= \begin{cases}1=g_{(1)}^{0} & i-\text { even } \\ g_{(1)} & i-\text { odd }\end{cases}
$$

Proof. It is easy to show (by direct differentiation and by induction) that if $g_{(1)}^{2}$ is given by Eq. (2.9) then

$$
g_{(2 n)}=A_{2 n}(g) ; \quad g_{(2 n+1)}=A_{2 n+1}(g) g_{(1)}
$$

where $A_{2 n}(g)$ and $A_{2 n+1}(g)$ are polynomials of $g$. 
Let then

$$
\Omega_{i}\left(g_{(1)}\right)= \begin{cases}1=g_{(1)}^{0} & i-\text { even } \\ g_{(1)} & i-\text { odd }\end{cases}
$$

Hence Eq.(2.10) can be written as

$$
g_{(i)}=A_{i}(g) \Omega_{i}\left(g_{(1)}\right)
$$

Thus

$$
g_{(i)}^{\lambda_{i}}=\left[A_{i}(g)\right]^{\lambda_{i}}\left[\Omega_{i}\left(g_{(1)}\right)\right]^{\lambda_{i}}
$$

Now from the definition of $\Omega_{i}$ - Eq.(2.11) we see that $\Omega_{i}^{\lambda_{i}}$ is equal to 1 if $i$ is even. $\Omega_{i}^{\lambda_{i}}$ is equal to $g_{(1)}^{\lambda_{i}}$ if $i$ is odd. But from Eq.(2.10) for the case of odd $\lambda_{i}$

$$
g_{(1)}^{\lambda_{i}}=g_{(1)}^{2\left(\left[\lambda_{i} / 2\right]\right)+1}=g_{(1)}\left(g_{(1)}^{2}\right)^{\left[\lambda_{i} / 2\right]}
$$

In addition from Eq.(2.10) it follows that $g_{(1)}^{2}$ is a polynomial of $g$ (this also can be seen from the definition of the simplest equation (2.9)). Thus we can define

$$
O_{i, \lambda_{i}}\left(g, g_{(1)}\right)= \begin{cases}1 & i-\text { even, } \lambda_{i} \text { - even } \\ \frac{1}{g_{(1)}} & i-\text { even, } \lambda_{i} \text { - odd } \\ \left(\sum_{j=0}^{m} a_{j} g^{j}\right)^{\left[\lambda_{i} / 2\right]} & i-\text { odd, } \lambda_{i} \text { - even } \\ \left(\sum_{j=0}^{m} a_{j} g^{j}\right)^{\left[\lambda_{i} / 2\right]} & i-\text { odd, } \lambda_{i} \text { - odd }\end{cases}
$$

and then

$$
\left[\Omega_{i}\left(g_{(1)}\right)\right]^{\lambda_{i}}=O_{i, \lambda_{i}}\left(g, g_{(1)}\right) \Omega_{\lambda_{i}}\left(g_{(1)}\right)
$$

Substitution of Eq.(2.16) in Eq.(2.13) leads us to the relationship we want to prove

$$
g_{(i)}^{\lambda_{i}}=\left[A_{i}(g)\right]^{\lambda_{i}} O_{i, \lambda_{i}}\left(g, g_{(1)}\right) \Omega_{\lambda_{i}}\left(g_{(1)}\right)
$$

Now we are in position to prove

Theorem. If $g_{(1)}^{2}$ is given by Eq.(2.9) and $f$ is a polynomial of $g$ given by Eq.(2.5) then for $h[f(g)]$ the following relationship holds

$$
h_{(n)}=K_{n}(q, m)(g)+g_{(1)} Z_{n}(q, m)(g)
$$

where $K_{n}(q, m)(g)$ and $Z_{n}(q, m)(g)$ are polynomials of the function $g(\xi)$. 
Proof. The substitution of Eqs. (2.17) and (2.7) in Eq.(2.3) leads to the following relationship for $h_{(n)}$

$$
h_{(n)}=\sum_{k=1}^{n} \sum_{r=0}^{q} \Theta_{r k} \frac{r !}{(r-k) !} b_{r} g^{r-k} \sum_{p(n, k)} n ! \prod_{i=1}^{n} \frac{\left[A_{i}(g)\right]^{\lambda_{i}} O_{i, \lambda_{i}}\left(g, g_{(1)}\right) \Omega_{\lambda_{i}}\left(g_{(1)}\right)}{\left(\lambda_{i} !\right)(i !)^{\lambda_{i}}}
$$

Now let us show that the relationship (2.18) can be written as

$$
h_{(n)}=K_{n}(q, m)(g)+g_{(1)} Z_{n}(q, m)(g)
$$

where $K_{n}(q, m)(g)$ and $Z_{n}(q, m)(g)$ are polynomials of the function $g(\xi)$. In oder to show this we rewrite Eq.(2.18) as

$h_{(n)}=\sum_{k=1}^{n} \sum_{r=0}^{q} \Theta_{r k} \frac{r !}{(r-k) !} b_{r} g^{r-k} \sum_{p(n, k)} n !\left[\prod_{i=1}^{n} \frac{\left[A_{i}(g)\right]^{\lambda_{i}}}{\left(\lambda_{i} !\right)(i !)^{\lambda_{i}}}\right] \prod_{i=1}^{n} O_{i, \lambda_{i}}\left(g, g_{(1)}\right) \Omega_{\lambda_{i}}\left(g_{(1)}\right)$

and consider $P=\prod_{i=1}^{n} O_{i, \lambda_{i}}\left(g, g_{(1)}\right) \Omega_{\lambda_{i}}\left(g_{(1)}\right)$. This product is equal of polynomial of $g$ multiplied by $g_{(1)}^{\sigma}$ where $\sigma$ is an integer. There are two possibilities for $\sigma$ :

- $\sigma$ : even. Then $\left(g_{(1)}^{2}\right)^{\sigma / 2}$ is a polynomial of $g$ according to Eq.(2.9)

- $\sigma$ : odd. Then $g_{(1)}^{\sigma}$ is equal to $g_{(1)}\left(g_{(1)}^{2}\right)^{[\sigma] / 2}$ and according to Eq.(2.9) this is equal to $g_{(1)}$ multiplied by a polynomial of $g$.

Because of all above there will be two kinds of terms in $h_{(n)}$ : terms that are polynomials of $g$ and terms that contain $g_{(1)}$ multiplied by a polynomial of g. Collecting the two kinds of terms we arrive at Eq.(2.19).

Let us note that for some values of $n$ one of the polynomials $K_{n}(q, m)$ or $Z_{n}(q, m)$ can be equal to 0 .

\subsection{Formulation of the method}

On the basis of all above the modified method of simplest equation based on simplest equation (2.9) can be formulated as follows:

1. We consider a nonlinear PDE $E^{*}\left(u(x, t), u_{x}(x, t), u_{t}(x, t), u_{x t}(x, t), \ldots\right)=$ 0 where $E^{*}$ is a polynomial of $u(x, t)$ and its derivatives. We search for solutions of this equation based on the ansatz $\xi=\mu x+\nu t$ where $\alpha$ and $\beta$ are parameters. 
2. The ansatz $\xi=\mu x+\nu t$ reduces the nonlinear PDE to the ODE $E\left(h, h_{(1)}, \ldots\right)=0$ where $E$ is a polynomial of $h$ and its derivatives.

3. We assume $h$ and $g$ are given by the Eqs. (2.5) and (2.9). Substitution of the relationships in the equation $E=0$ reduces any derivative of this equation to a term of the kind (2.19).

4. As the terms in $E=0$ are polynomials of $h$ and its derivatives then the equation reduces to a polynomial containing $g, g_{(1)}, g_{(1)}^{2}, \ldots: E=$ $W_{0}^{*}(g)+W_{1}^{*}(g) g_{(1)}+W_{2}^{*}(g) g_{(1)}^{2}+\ldots$ Eq.(2.9) reduces the higher degrees $(n>1)$ of $g_{(1)}$ to a polynomial of $g$ (for even $n$ ) or to a polynomial of $g$ multiplied by $g_{(1)}$ (for odd $n$ ). Thus equation $E=0$ is reduces to

$$
E=W_{0}(g)+W_{1}(g) g_{(1)}=0
$$

where $W_{0,1}(g)$ are polynomials of $g$.

5. In order to obtain a nontrivial solution of Eq.(2.21) we have to balance the highest degree of the polynomial $W_{0}$ (i.e., to ensure that there are at least two terms that contain the highest degree of $W_{0}$ ). The same has to be made for the polynomial $W_{1}$. As a result we obtain one or two relationships among the parameters of the equation and parameters of the solution. These equations are called balance equations. Balance equations fix parameters $q$ and $m$.

6. Further we set to 0 all coefficients of the polynomials $W_{0}(g)$ and $W_{1}(g)$. The result is a system of nonlinear algebraic equations that contains the parameters of the equation, parameters of the solution (2.5) and parameters of the simplest equation (2.9).

7. Any nontrivial solution of the above system of algebraic equations (if it exists) leads to a traveling wave solution of the nonlinear PDE $E^{*}=0$.

\section{Calculation of some of polynomials $K_{n}$ and $Z_{n}$ from Eq.(2.19)}

The derivatives $h_{(1)}, h_{(2)}, h_{(3)}, h_{(4)}, h_{(5)}, h_{(6)}$ and $h_{(7)}$ are much used in the model nonlinear partial differential equations. Below we shall calculate the polynomials $K_{n}$ and $Z_{n}$ connected to these derivatives.

Let us mention first that the polynomials $K_{n+1}$ and $Z_{n+1}$ are connected to the polynomials $K_{n}$ and $Z_{n}$. This relationship can be obtained on the 
basis of the relationship

$$
h_{(n+1)}=\frac{d}{d \xi} h_{(n)}
$$

Substitution of Eq.(2.19) in Eq.(3.1) leads to

$$
h_{(n+1)}=\left[Z_{n} g_{(2)}+\frac{d Z_{n}}{d g} g_{(1)}^{2}\right]+\frac{d K_{n}}{d g} g_{(1)}
$$

Taking in account Eq.(2.9) we obtain

$$
\begin{aligned}
K_{n+1} & =\frac{Z_{n}}{2} \sum_{j=0}^{m} j a_{j} g^{j-1}+\frac{d Z_{n}}{d g} \sum_{j=0}^{m} a_{j} g^{j} \\
Z_{n+1} & =\frac{d K_{n}}{d g}
\end{aligned}
$$

Hence we need to calculate only $K_{1}$ and $Z_{1}$ and then we can obtain $K_{n}$ and $Z_{n}, n=2,3, \ldots$ by the recurrence equations (3.3).

We can write

$$
\begin{aligned}
K_{0} & =\sum_{r=0}^{q} b_{r} g^{r} \\
Z_{0} & =0
\end{aligned}
$$

From Eq.(2.18) we obtain

$$
K_{1}=0 ; \quad Z_{1}=\sum_{r=0}^{q} r b_{r} g^{r-1}
$$

Then

$$
\begin{aligned}
K_{2} & =\sum_{r=0}^{q} \sum_{j=0}^{m}\left[\frac{1}{2} j r+r(r-1)\right] a_{j} b_{r} g^{j+r-2} \\
Z_{2} & =0 \\
K_{3} & =0 ; \\
Z_{3} & =\sum_{r=0}^{q} \sum_{j=0}^{m}\left[\frac{1}{2} j r+r(r-1)\right](j+r-2) a_{j} b_{r} g^{j+r-3}
\end{aligned}
$$




$$
\begin{aligned}
K_{4}= & \sum_{r=0}^{q} \sum_{j=0}^{m} \sum_{u=0}^{m}\left[\left(\frac{1}{2} j r+r(r-1)\right)(j+r-2)\left(\frac{1}{2} u+j+r-3\right)\right] a_{j} b_{r} a_{u} g^{j+r+u-4} \\
Z_{4}= & 0 \\
K_{5}= & 0 ; \\
Z_{5}= & \sum_{r=0}^{q} \sum_{j=0}^{m} \sum_{u=0}^{m}\left[\left(\frac{1}{2} j r+r(r-1)\right)(j+r-2)\left(\frac{1}{2} u+j+r-3\right)\right](j+ \\
& r+u-4) a_{j} b_{r} a_{u} g^{j+r+u-5} \\
K_{6}= & \sum_{r=0}^{q} \sum_{j=0}^{m} \sum_{u=0}^{m} \sum_{v=0}^{m}\left[\left(\frac{1}{2} j r+r(r-1)\right)(j+r-2)\left(\frac{1}{2} u+j+r-3\right)(j+\right. \\
& r+u-4)]\left(\frac{1}{2} v+j+r+u-5\right) a_{j} b_{r} a_{u} a_{v} g^{j+r+u+v-6} \\
Z_{6}= & 0 . \\
K_{7}= & 0 ; \\
Z_{7}= & \sum_{r=0}^{q} \sum_{j=0}^{m} \sum_{u=0}^{m} \sum_{v=0}^{m}\left[\left(\frac{1}{2} j r+r(r-1)\right)(j+r-2)\left(\frac{1}{2} u+j+r-3\right)(j+\right. \\
& r+u-4)]\left(\frac{1}{2} v+j+r+u-5\right)(j+r+u+v-6) a_{j} b_{r} a_{u} a_{v} g^{j+r+u+v-7} \\
&
\end{aligned}
$$

etc.

For the practical application of the modified method of simplest equation we need to calculate the maximum grade of polynomials in $h_{(n)}$. As we have seen above in the text $h_{(n)}$ consists of two kinds of terms: polynomial of $g$ plus another polynomial of $g$ multiplied by $g_{(1)}$. We note that the above maximum grades have to be non-negative. Thus the obtained relationships below hold when the corresponding maximum degree is $\max \geq 0$.

By the method of mathematical induction we can prove that:

- For $h_{(2 \sigma)}$ : Maximum grade of polynomial $K_{2 \sigma}(g)$ is $\max =q+\sigma(m-2)$ where $\sigma=1,2, \ldots$ Maximum grade of the polynomial $Z_{2 \sigma}(g)$ is 0 .

- For $h_{(2 \sigma+1)}$ : Maximum grade of polynomial of $K_{2 \sigma+1}(g)$ is 0 . Maximum grade of the polynomial of $Z_{2 \sigma+1}(g)$ is $\max =q+\sigma(m-2)-1$ where $\sigma=0,1, \ldots$ 


\section{Examples}

\subsection{Generalized Korteweg-deVries equation}

We shall consider the equation

$$
\frac{\partial u}{\partial t}+A u^{p} \frac{\partial u}{\partial x}+\frac{\partial^{3} u}{\partial x^{3}}=0
$$

where $A$ is a parameter and $p$ is a positive integer number. This equation has different applications as for an example in the electrohydrodynamics [53]. For the case when $p$ is a positive integer Eq.(3.1) is called generalized KortewegdeVries equation. It is obtained by addition of the dispersion term $\frac{\partial^{3} u}{\partial x^{3}}$ to the nonlinear convective wave equation $\frac{\partial u}{\partial t}+A u^{p} \frac{\partial u}{\partial x}=0$.

We search for solutions of Eq.(4.1) of the kind $u=h[f(g(\xi))]$ where $\xi=\alpha x+\beta t, g$ is solution of the simplest equation (2.9) and $f$ is given by Eq.(2.5). The substitution of $u=h[f(g(\xi))]$ in Eq.(4.1) leads to equation of the kind (2.21) where

$$
\begin{aligned}
& W_{0}(g)=\nu K_{1}(g)+\mu A K_{0}(g)^{p} K_{1}(g)+\mu^{3} K_{3}(g) \\
& W_{1}(g)=\nu Z_{1}(g)+\mu A K_{0}(g)^{p} Z_{1}(g)+\mu^{3} Z_{3}(g)
\end{aligned}
$$

As $K_{1}=K_{3}=0$ there is no need to balance the relationship for $W_{0}(g)$. The relationship for $W_{1}(g)$ has to be balanced however. The resulting balance equation is

$$
m=2+p q
$$

Let us consider the case $q=1, m=2+p$. Then from Eqs.(2.5) and (2.9) one obtains

$$
h=b_{0}+b_{1} g ; \quad g_{(1)}^{2}=\sum_{j=0}^{2+p} a_{j} g^{j} .
$$

Substituting corresponding relationships for the polynomials $Z_{1}, Z_{3}$ and $K_{0}$ in the second of equations (4.2) we obtain the following system of nonlinear algebraic relationships among the parameters of Eq.(4.1) and the parameters of the solution:

$$
\nu b_{1} \delta_{0, k}+\left(\begin{array}{l}
p \\
k
\end{array}\right) \mu A b_{0}^{p-k} b_{1}^{k+1}+\frac{1}{2} \mu^{3}(k+1)(k+2) a_{k+2} b_{1}=0, \quad k=0, \ldots, p .
$$

where $\delta$ is the delta-symbol of Kronecker. 
Solution of Eqs.(4.5) can be obtained when $b_{0}=0$. Then

$$
\nu b_{1} \delta_{0, k}+\left(\begin{array}{l}
p \\
k
\end{array}\right) \mu A b_{1}^{k+1} \delta_{k, p}+\frac{1}{2} \mu^{3}(k+1)(k+2) a_{k+2} b_{1}=0, \quad k=0, \ldots, p .
$$

The system (4.6) is reduced to

$$
\begin{aligned}
k= & 0: \quad \nu+\mu^{3} a_{2}=0 \\
k= & 1: \quad a_{3}=0 \\
& \cdots \cdots \\
k= & p-1: \quad a_{p+1}=0 \\
k= & p: \quad A b_{1}^{p}+\frac{1}{2} \mu^{2}(p+1)(p+2) a_{p+2}=0 .
\end{aligned}
$$

The system (4.7) has a solution:

$$
a_{2}=-\frac{\nu}{\mu^{3}} ; \quad a_{p+2}=-\frac{2 A b_{1}^{p}}{\mu^{2}(p+1)(p+2)}
$$

Hence the simplest equation Eq.(2.9) becomes

$$
g_{(1)}^{2}=-\frac{\nu}{\mu^{3} A} g^{2}-\frac{2 b_{1}^{p}}{\mu^{2} A(p+1)(p+2)} g^{p+2}
$$

A solution of this simplest equation is as follows. The function

$$
g(\xi)=\frac{\Omega}{\cosh ^{\omega}(\xi)}
$$

(where $\omega$ and $\Omega$ are parameters) is solution of the equation

$$
g_{(1)}^{2}=\omega^{2} g^{2}-\frac{\omega^{2}}{\Omega^{2 / \omega}} g^{2+2 / \omega}
$$

Hence if

$$
\omega=\frac{2}{p} ; \quad \mu^{2}=\frac{p^{2} A \Omega^{p} b_{1}^{p}}{2(p+1)(p+2)} ; \quad \nu=-\frac{4 \mu^{3}}{p^{2}}
$$

then

$$
u(\xi)=\frac{\Omega b_{1}}{\cosh ^{2 / p}(\xi)} ; \xi=\mu x+\nu t
$$

is solution of Eq.(4.1).

Let us note that according to Eqs.(4.10) and (4.11)

$$
V_{0,0,4 / p^{2}, 0, \ldots, a_{p+2}=-\frac{4}{p^{2} \Omega^{p}}}(\xi ; 1,2,2+p)=\frac{\Omega}{\cosh ^{2 / p}(\xi)}
$$


Let us consider the particular case $p=1, A=-6$. Then Eq.(4.1) is reduced to the classical Korteweg-deVries equation. Setting $b_{1}=1$ and $\Omega=-2$ we obtain $\mu=1, \nu=-4$. Thus the solution (4.13) reduces to the one-soliton solution of the equation of Korteweg-deVries

$$
u(x, t)=-\frac{2}{\cosh ^{2}(x-4 t)}
$$

Let us stress the following. Above $p$ was arbitrary non-negative integer. Now we shall show that $p$ can be arbitrary non-zero real number. Namely we shall prove

Proposition. Eq. (4.13) is solution of Eq. (4.1) for arbitrary real nonzero value of $p$.

Proof. Let us substitute Eq.(4.13) in Eq.(4.1) where parameters $\alpha$ and $\beta$ are given by Eq.(4.12) and $p$ is an arbitrary nonzero real number. Eq.(4.1) is satisfied. Hence Eq.(4.13) is solution of Eq.(4.1) for arbitrary real $p \neq 0$.

Let for an example $p=3 / 2$. Then a solitary wave solution of the equation

$$
\frac{\partial u}{\partial t}+A u^{3 / 2} \frac{\partial u}{\partial x}+\frac{\partial^{3} u}{\partial x^{3}}=0
$$

is

$$
u(x, t)=\Omega b_{1} \cosh ^{-4 / 3}\left[\left(\frac{9}{70} A \Omega^{3 / 2} b_{1}^{3 / 2}\right)^{1 / 2} x-\frac{16}{9}\left(\frac{9}{70} A \Omega^{3 / 2} b_{1}^{3 / 2}\right)^{3 / 2} t\right]
$$

\subsection{Application of methodology to the higher order Korteweg-deVries equation}

Let us apply the above methodology to a more complicated equation such as the second-order Korteweg-deVries equation

$$
\frac{\partial u}{\partial t}+\frac{\partial u}{\partial x}+\alpha_{0} u \frac{\partial u}{\partial x}+\alpha_{1} \frac{\partial u}{\partial x} \frac{\partial^{2} u}{\partial x^{2}}+\alpha_{2} u \frac{\partial^{3} u}{\partial x^{3}}+\alpha_{3} u^{2} \frac{\partial u}{\partial x}+\alpha_{4} \frac{\partial^{3} u}{\partial x^{3}}+\alpha_{5} \frac{\partial^{5} u}{\partial x^{5}}=0
$$

This equation is known also as Olver equation [54] and it is a second order equation for description of shallow water waves (the first order equation is the famous Korteweg-deVries equation). Eq.(4.18) has been used also as a model equation for nonlinear waves in a liquid with gas bubbles [55, 56] From the point of view of the method of simplest equation (based on the 
first step of a test for Painleve property) the equation was discussed in [55]57] and various solutions expressed by the elliptic function of Weierstrass have been obtained there. Below we shall discuss Eq.(4.18) from the point of view of the modified method of simplest equation (based on the concept for balance equation). We stress again that the modified method of simplest equation is a version of the method of simplest equation and then some of the obtained below solutions (especially for the case $m=3$ ) will be the same as these obtained in [57]. After studying the methodologies from [57] and from this paper the reader will have an extensive understanding about the possibilities for obtaining exact analytical solutions of nonlinear partial differential equations on the basis of the method of simplest equation.

We search for a solution of the kind $u(x, t)=u(\xi)=u(\alpha x+\beta t)$ (We note that in [57] $\alpha=1$ ). According to the theory above Eq.(4.18) can be reduced to the following system of equations

$$
\begin{aligned}
W_{0}(g)= & 0 ; \\
W_{1}(g)= & (\mu+\nu) Z_{1}(g)+\alpha_{0} \mu K_{0}(g) Z_{1}(g)+\alpha_{1} \mu^{3} K_{2}(g) Z_{1}(g)+ \\
& \alpha_{2} \mu^{3} K_{0}(g) Z_{3}(g)+\alpha_{3} \mu K_{0}^{2}(g) Z_{1}(g)+\alpha_{4} \mu^{3} Z_{3}(g)+ \\
& \alpha_{5} \mu^{5} Z_{5}(g)=0
\end{aligned}
$$

The second of Eqs.(4.19) has to be balanced and the balance is as follows

1. $m=1$ : there is no balance;

2. $m=2$ : there is no balance;

3. $m \geq 3$ : the equation is balanced if $q=m-2$.

Let us now consider several cases.

\subsection{Case $m=3$}

In this case $q=1$. Then

$$
u(\xi)=b_{0}+b_{1} g(\xi) ; \quad \xi=\mu x+\nu t
$$

and

$$
g_{(1)}^{2}=a_{0}+a_{1} g+a_{2} g^{2}+a_{3} g^{3}
$$

In addition we have to solve the system of nonlinear algebraic equations for the parameters of the solution that can be obtained from the equation $W_{1}(g)=0$ from Eqs.(4.19). 
We note that the general solution of Eq.4.21) is given by the special function $V_{a_{0}, a_{1}, a_{2}, a_{3}}(\xi ; 1,2,3)$ and for the special case when $a_{2}=0$ and $a_{3}=4$ we have reduction of $V$ to the elliptic function of Weierstrass

$$
V_{a_{0}, a_{1}, 0,4}(\xi ; 1,2,3)=\wp\left(\xi ; a_{0}, a_{1}\right)
$$

Let us first consider the case of general non-reduced solution $V_{a_{0}, a_{1}, a_{2}, a_{3}}(\xi ; 1,2,3)$ of Eq.(4.21). The system of nonlinear algebraic equations arising from second of the Eqs.(4.19) is (A.1) from the appendix A. The solution of the system (A.1) is:

$$
\begin{aligned}
a_{3}= & \frac{b_{1}}{30 \mu^{2} \alpha_{5}}\left(-\alpha_{1}-2 \alpha_{2}+\sqrt{\left(\alpha_{1}+2 \alpha_{2}\right)^{2}-40 \alpha_{3} \alpha_{5}}\right) \\
a_{2}= & -\frac{1}{5 \mu^{2} \alpha_{5}\left(\alpha_{1}+\sqrt{\left(\alpha_{1}+2 \alpha_{2}\right)^{2}-40 \alpha_{3} \alpha_{5}}\right)}[ \\
& \left(\alpha_{2} b_{0}+\alpha_{4}\right) \sqrt{\left(\alpha_{1}+2 \alpha_{2}\right)^{2}-40 \alpha_{3} \alpha_{5}}-\alpha_{1} \alpha_{2} b_{0}-2 \alpha_{2}^{2} b_{0}+ \\
& \left.20 \alpha_{3} \alpha_{5} b_{0}+10 \alpha_{0} \alpha_{5}-\alpha_{4} \alpha_{1}-2 \alpha_{4} \alpha_{2}\right] \\
a_{1}= & \frac{T_{1}}{T_{2}} \\
T_{1}= & -\frac{2}{5}\left[25 \mu \alpha_{1}^{2} \alpha_{3} \alpha_{5} b_{0}^{2}-6 \mu \alpha_{2}^{4} b_{0}^{2}+90 \mu \alpha_{2}^{2} \alpha_{3} \alpha_{5} b_{0}^{2}+25 \mu \alpha_{0} \alpha_{1}^{2} \alpha_{5} b_{0}+\right. \\
& 30 \mu \alpha_{0} \alpha_{2}^{2} \alpha_{5} b_{0}-300 \mu \alpha_{0} \alpha_{3} \alpha_{5}^{2} b_{0}+2 \mu \alpha_{1}^{2} \alpha_{2} \alpha_{4} b_{0}-2 \mu \alpha_{1} \alpha_{2}^{2} \alpha_{4} b_{0}+ \\
& 50 \mu \alpha_{0}^{2} \alpha_{5}^{2}-35 \mu \alpha_{0} \alpha_{1} \alpha_{4} \alpha_{5}-20 \mu \alpha_{0} \alpha_{2} \alpha_{4} \alpha_{5}+\mu \alpha_{1}^{2} \alpha_{4}^{2}-6 \mu \alpha_{2}^{2} \alpha_{4}^{2}+ \\
& 25 \mu \alpha_{1}^{2} \alpha_{5}+50 \mu \alpha_{2}^{2} \alpha_{5}-500 \mu \alpha_{3} \alpha_{5}^{2}+25 \nu \alpha_{1}^{2} \alpha_{5}+50 \nu \alpha_{2}^{2} \alpha_{5}- \\
& 500 \nu \alpha_{3} \alpha_{5}^{2}+80 \mu \alpha_{3} \alpha_{4}^{2} \alpha_{5}+50 \mu \alpha_{1} \alpha_{2} \alpha_{5}+50 \nu \alpha_{1} \alpha_{2} \alpha_{5}+\mu \alpha_{1}^{2} \alpha_{2}^{2} b_{0}^{2}- \\
& \mu \alpha_{1} \alpha_{2}^{3} b_{0}^{2}-300 \mu \alpha_{3}^{2} \alpha_{5}^{2} b_{0}^{2}-12 \mu \alpha_{2}^{3} \alpha_{4} b_{0}-\mu \alpha_{1} \alpha_{2} \alpha_{4}^{2}-20 \mu \alpha_{1} \alpha_{2} \alpha_{3} \alpha_{5} b_{0}^{2}+ \\
& 15 \mu \alpha_{0} \alpha_{1} \alpha_{2} \alpha_{5} b_{0}-70 \mu \alpha_{1} \alpha_{3} \alpha_{4} \alpha_{5} b_{0}+120 \mu \alpha_{2} \alpha_{3} \alpha_{4} \alpha_{5} b_{0}+ \\
& \left(6 \mu \alpha_{2}^{2} \alpha_{4} b_{0}-\mu \alpha_{1} \alpha_{2}^{2} b_{0}^{2}-15 \mu \alpha_{0} \alpha_{4} \alpha_{5}+3 \mu \alpha_{2}^{3} b_{0}^{2}-\mu a_{1} a_{4}^{2}+3 \mu a_{2} a_{4}^{2}+\right. \\
T_{2}= & \mu^{2} \alpha_{5} b_{1}\left[\left(5 \alpha_{1}^{2}+3 \alpha-2^{2}-30 \alpha_{3} \alpha_{5}\right) \sqrt{\left(\alpha_{1}+2 \alpha_{2}\right)^{2}-40 \alpha_{3} \alpha_{5}}+\right. \\
& \left.10 \alpha_{1}^{2} \alpha_{2}+7 \alpha_{1} \alpha_{2}^{2}-130 \alpha_{1} \alpha_{3} \alpha_{5}-6 \alpha_{2}^{3}+60 \alpha_{2} \alpha_{3} \alpha_{5}\right] \\
& 25 \mu \alpha_{1} \alpha_{5}+25 \nu \alpha_{1} \alpha_{5}+25 \mu \alpha_{1} \alpha_{3} \alpha_{5} b_{0}^{2}-30 \mu \alpha_{2} \alpha_{3} \alpha_{5} b_{0}^{2}+25 \mu \alpha_{0} \alpha_{1} \alpha_{5} b_{0}- \\
& \left.\left.2 \mu \alpha_{1} \alpha_{2} \alpha_{4} b_{0}-30 \mu \alpha_{3} \alpha_{4} \alpha_{5} b_{0}\right) \sqrt{\left(\alpha_{1}+2 \alpha_{2}\right)^{2}-40 \alpha_{3} \alpha_{5}}\right] \\
& \\
& \\
&
\end{aligned}
$$


The solution of the higher order Korteweg-deVries equation (4.18) is

$$
u(\xi)=b_{0}+b_{1} V_{a_{0}, a_{1}, a_{2}, a_{3}}(\xi ; 1,2,3) ; \quad \xi=\mu x+\nu t
$$

where $a_{1,2,3}$ are given by Eqs.(4.23).

Let us now consider the particular case (4.22) when the V-function is reduced to the elliptic function of Weierstrass. In this case we have to set $a_{2}=0 ; a_{3}=4$ in the system (??). The solution of the obtained system of algebraic equations is

$$
\begin{aligned}
b_{0} & =-\frac{\alpha_{0} \sqrt{\left(\alpha_{1}+\alpha_{2}\right)^{2}-40 \alpha_{3} \alpha_{5}}-\alpha_{0} \alpha_{1}-2 \alpha_{0} \alpha_{2}+4 \alpha_{4} \alpha_{3}}{2 \alpha_{3}\left(\sqrt{\left(\alpha_{1}+2 \alpha_{2}\right)^{2}-40 \alpha_{3} \alpha_{5}}-\alpha_{1}\right)} \\
b_{1} & =\frac{3 \mu^{2}\left(-\alpha_{1}-2 \alpha_{2}+\sqrt{\left(\alpha_{1}+2 \alpha_{2}\right)^{2}-40 \alpha_{3} \alpha_{5}}\right)}{\alpha_{3}}
\end{aligned}
$$

$$
\begin{aligned}
a_{1}= & \frac{T_{3}}{T_{4}} \\
T_{3}= & -\frac{1}{3}\left[-\mu \alpha_{0}^{2} \alpha_{1}^{2}-2 \mu \alpha_{0}^{2} \alpha_{1} \alpha_{2}+20 \mu \alpha_{0}^{2} \alpha_{3} \alpha_{5}-8 \mu \alpha_{0} \alpha_{2} \alpha_{3} \alpha_{4}+8 \mu \alpha_{3}^{2} \alpha_{4}^{2}+\right. \\
& +4 \mu \alpha_{1}^{2} \alpha_{3}+8 \mu \alpha_{1} \alpha_{2} \alpha_{3}+8 \mu \alpha_{2}^{2} \alpha_{3}-80 \mu \alpha_{3}^{2} \alpha_{5}+4 \nu \alpha_{1}^{2} \alpha_{3}+8 \nu \alpha_{1} \alpha_{2} \alpha_{3}+ \\
& \left.8 \nu \alpha_{2}^{2} \alpha_{3}-80 \nu \alpha_{3}^{2} \alpha_{5}+\left(\mu \alpha_{0}^{2} \alpha_{1}-4 \mu \alpha_{1} \alpha_{3}-4 \nu \alpha_{1} \alpha_{3}\right) \sqrt{\left(\alpha_{1}+2 \alpha_{2}\right)^{2}-40 \alpha_{3} \alpha_{5}}\right] \\
T_{4}= & \mu^{5}\left(\sqrt{\left(\alpha_{1}+2 \alpha_{2}\right)^{2}-40 \alpha_{3} \alpha_{5}}-\alpha_{1}\right)^{2}\left(\alpha_{1} \sqrt{\left(\alpha_{1}+2 \alpha_{2}\right)^{2}-40 \alpha_{3} \alpha_{5}}-\right. \\
& \left.\alpha_{1}^{2}-2 \alpha_{1} \alpha_{2}+12 \alpha_{3} \alpha_{5}\right)
\end{aligned}
$$

The solution of Eq.(4.18) becomes

$$
\begin{aligned}
u(\xi)= & -\frac{\alpha_{0} \sqrt{\left(\alpha_{1}+2 \alpha_{2}\right)^{2}-40 \alpha_{3} \alpha_{5}}-\alpha_{0} \alpha_{1}-2 \alpha_{0} \alpha_{2}+4 \alpha_{4} \alpha_{3}}{2 \alpha_{3}\left(\sqrt{\left(\alpha_{1}+2 \alpha_{2}\right)^{2}-40 \alpha_{3} \alpha_{5}}-\alpha_{1}\right)}+ \\
& \frac{3 \mu^{2}\left(-\alpha_{1}-2 \alpha_{2}+\sqrt{\left(\alpha_{1}+2 \alpha_{2}\right)^{2}-40 \alpha_{3} \alpha_{5}}\right)}{\alpha_{3}} \wp\left(\xi ; a_{0}, a_{1}\right) \\
& \xi=\mu x+\nu t .
\end{aligned}
$$


where $a_{1}$ is given by the corresponding relationship from Eqs. (4.25).

Let us now consider as simplest equation Eq.(4.11) for the case $\omega=2$, namely

$$
g_{(1)}^{2}=4 g^{2}-\frac{4}{\Omega} g^{3}
$$

In this case $a_{0}=a_{1}=0, a_{2}=4$ and $a_{3}=-\frac{4}{\Omega}$. The solution of Eq.(4.27) is

$$
g(\xi)=\frac{\Omega}{\cosh ^{2}(\xi)}=V_{0,0,4,-\frac{4}{\Omega}}(\xi ; 1,2,3)
$$

One solution of the system of nonlinear algebraic equations for this case is

$$
\begin{aligned}
b_{0}= & -\frac{1}{\alpha_{3} \sqrt{\left(\alpha_{1}+2 \alpha_{2}\right)^{2}-40 \alpha_{3} \alpha_{5}}+\alpha_{1}}\left[\left(4 \mu^{2} \alpha_{1}+4 \mu^{2} \alpha_{2}+\right.\right. \\
& \left.\alpha_{0}\right) \sqrt{\left(\alpha_{1}+2 \alpha_{2}\right)^{2}-40 \alpha_{3} \alpha_{5}}+4 \mu^{2} \alpha_{1}^{2}+12 \mu^{2} \alpha_{1} \alpha_{2}+ \\
& \left.8 \mu^{2} \alpha_{2}^{2}-80 \alpha_{5} \mu^{2} \alpha_{3}+\alpha_{0} \alpha_{1}+2 \alpha_{0} \alpha_{2}-4 \alpha_{4} \alpha_{3}\right]
\end{aligned}
$$

$$
\begin{aligned}
b_{1}= & \frac{3 \mu^{2}}{\alpha_{3} \Omega}\left(\alpha_{1}+2 \alpha_{2}+\sqrt{\left(\alpha_{1}+2 \alpha_{2}\right)^{2}-40 \alpha_{3} \alpha_{5}}\right) \\
\nu= & -\frac{\mu}{4 \alpha_{3}\left(\sqrt{\left(\alpha_{1}+2 \alpha_{2}\right)^{2}-40 \alpha_{3} \alpha_{5}} \alpha_{1}+\alpha_{1}^{2}+2 \alpha_{1} \alpha_{2}+2 \alpha_{2}^{2}-20 \alpha_{3} \alpha_{5}\right)}[ \\
& \left(\alpha_{0}^{2} \alpha_{1}+4 \alpha_{1} \alpha_{3}+16 \mu^{4} \alpha_{1}^{3}+32 \mu^{4} \alpha_{1}^{2} \alpha_{2}+16 \mu^{4} \alpha_{1} \alpha_{2}^{2}-\right. \\
& \left.256 \mu^{4} \alpha_{1} \alpha_{3} \alpha_{5}\right) \sqrt{\left(\alpha_{1}+2 \alpha_{2}\right)^{2}-40 \alpha_{3} \alpha_{5}}+64 \mu^{4} \alpha_{1}^{3} \alpha_{2}+80 \mu^{4} \alpha_{1}^{2} \alpha_{2}^{2}- \\
& 576 \mu^{4} \alpha_{1}^{2} \alpha_{3} \alpha_{5}+32 \mu^{4} \alpha_{1} \alpha_{2}^{3}-512 \mu^{4} \alpha_{1} \alpha_{2} \alpha_{3} \alpha_{5}-192 \mu^{4} \alpha_{2}^{2} \alpha_{3} \alpha_{5}+1920 \mu^{4} \alpha_{3}^{2} \alpha_{5}^{2}- \\
& \alpha_{0}^{2} \alpha_{1}^{2}-2 \alpha_{0}^{2} \alpha_{1} \alpha_{2}+20 \alpha_{0}^{2} \alpha_{3} \alpha_{5}-8 \alpha_{0} \alpha_{2} \alpha_{3} \alpha_{4}+8 \alpha_{3}^{2} \alpha_{4}^{2}+4 \alpha_{1}^{2} \alpha_{3}+8 \alpha_{1} \alpha_{2} \alpha_{3}+ \\
& \left.8 \alpha_{2}^{2} \alpha_{3}-80 \alpha_{3}^{2} \alpha_{5}\right]
\end{aligned}
$$

and the corresponding solitary-wave solution of Eq.(4.18) is

$$
\begin{aligned}
u(\xi)= & -\frac{1}{\alpha_{3} \sqrt{\left(\alpha_{1}+2 \alpha_{2}\right)^{2}-40 \alpha_{3} \alpha_{5}}+\alpha_{1}}\left[\left(4 \mu^{2} \alpha_{1}+4 \mu^{2} \alpha_{2}+\right.\right. \\
& \left.\alpha_{0}\right) \sqrt{\left(\alpha_{1}+2 \alpha_{2}\right)^{2}-40 \alpha_{3} \alpha_{5}}+4 \mu^{2} \alpha_{1}^{2}+12 \mu^{2} \alpha_{1} \alpha_{2}+ \\
& \left.8 \mu^{2} \alpha_{2}^{2}-80 \alpha_{5} \mu^{2} \alpha_{3}+\alpha_{0} \alpha_{1}+2 \alpha_{0} \alpha_{2}-4 \alpha_{4} \alpha_{3}\right]+\frac{3 \mu^{2}}{\alpha_{3}}\left(\alpha_{1}+2 \alpha_{2}+\right. \\
& \left.\sqrt{\left(\alpha_{1}+2 \alpha_{2}\right)^{2}-40 \alpha_{3} \alpha_{5}}\right) \frac{1}{\cosh ^{2}(\xi)}
\end{aligned}
$$


where

$$
\begin{aligned}
\xi= & \mu x-\frac{\mu}{4 \alpha_{3}\left(\sqrt{\left(\alpha_{1}+2 \alpha_{2}\right)^{2}-40 \alpha_{3} \alpha_{5}} \alpha_{1}+\alpha_{1}^{2}+2 \alpha_{1} \alpha_{2}+2 \alpha_{2}^{2}-20 \alpha_{3} \alpha_{5}\right)}[ \\
& \left(\alpha_{0}^{2} \alpha_{1}+4 \alpha_{1} \alpha_{3}+16 \mu^{4} \alpha_{1}^{3}+32 \mu^{4} \alpha_{1}^{2} \alpha_{2}+16 \mu^{4} \alpha_{1} \alpha_{2}^{2}-\right. \\
& \left.256 \mu^{4} \alpha_{1} \alpha_{3} \alpha_{5}\right) \sqrt{\left(\alpha_{1}+2 \alpha_{2}\right)^{2}-40 \alpha_{3} \alpha_{5}}+64 \mu^{4} \alpha_{1}^{3} \alpha_{2}+80 \mu^{4} \alpha_{1}^{2} \alpha_{2}^{2}- \\
& 576 \mu^{4} \alpha_{1}^{2} \alpha_{3} \alpha_{5}+32 \mu^{4} \alpha_{1} \alpha_{2}^{3}-512 \mu^{4} \alpha_{1} \alpha_{2} \alpha_{3} \alpha_{5}-192 \mu^{4} \alpha_{2}^{2} \alpha_{3} \alpha_{5}+1920 \mu^{4} \alpha_{3}^{2} \alpha_{5}^{2}- \\
& \alpha_{0}^{2} \alpha_{1}^{2}-2 \alpha_{0}^{2} \alpha_{1} \alpha_{2}+20 \alpha_{0}^{2} \alpha_{3} \alpha_{5}-8 \alpha_{0} \alpha_{2} \alpha_{3} \alpha_{4}+8 \alpha_{3}^{2} \alpha_{4}^{2}+4 \alpha_{1}^{2} \alpha_{3}+8 \alpha_{1} \alpha_{2} \alpha_{3}+ \\
& \left.8 \alpha_{2}^{2} \alpha_{3}-80 \alpha_{3}^{2} \alpha_{5}\right] t
\end{aligned}
$$

\subsection{Case $m=4$}

In this case $q=2$. Then

$$
u(\xi)=b_{0}+b_{1} g(\xi)+b_{2} g^{2}(\xi) ; \quad \xi=\mu x+\nu t
$$

and

$$
g_{(1)}^{2}=a_{0}+a_{1} g+a_{2} g^{2}+a_{3} g^{3}+a_{4} g^{4}
$$

In addition we have to solve the system of nonlinear algebraic equations for the parameters of the solution that can be obtained from the equation $W_{1}(g)=0$ from Eqs.(4.19).

The general solution of Eq..(4.32) is given by the special function $V_{a_{0}, a_{1}, a_{2}, a_{3}, a_{4}}(\xi ; 1,2,4)$. For several particular cases this function can be reduced to the elliptic functions of Jacobi. Examples are

$$
\begin{aligned}
V_{1,0,-\left(1+k^{2}\right), 0, k^{2}}(\xi ; 1,2,4) & =\operatorname{sn}(\xi ; k) \\
V_{1-k^{2}, 0,\left(2 k^{2}-1\right), 0,-k^{2}}(\xi ; 1,2,4) & =\operatorname{cn}(\xi ; k) \\
V_{-\left(1-k^{2}\right), 0,\left(2-k^{2}\right), 0,-1}(\xi ; 1,2,4) & =\operatorname{dn}(\xi ; k) .
\end{aligned}
$$

For the general case the system of nonlinear algebraic equations becomes (A.2) from the Appendix. This system of equations possesses exact solutions for $a_{0}, a_{1}, a_{2}, a_{3}, a_{4}$ but they are very long and we shall not reproduce them here. Instead of this we shall give a solution for an illustrative particular case. From the first of equations (A.2) one obtains a solution as follows

$$
a_{4}=\frac{b_{2}\left[-\alpha_{1}-2 \alpha_{2}+\sqrt{\left(\alpha_{1}+2 \alpha_{2}\right)^{2}-40 \alpha_{3} \alpha_{5}}\right]}{120 \alpha_{5} \mu^{2}}
$$

Let us consider the particular case

$$
\alpha_{5}=\frac{\left(\alpha_{1}+2 \alpha_{2}\right)^{2}}{40 \alpha_{3}}
$$


For this particular case a solution of the system of equations (A.2) is as follows

$$
\begin{aligned}
a_{4}= & -\frac{\alpha_{3} b_{2}}{3 \mu^{2}\left(\alpha_{1}^{2}+2 \alpha_{2}\right)} \\
a_{3}= & -\frac{2 \alpha_{3} b_{1}}{3 \mu^{2}\left(\alpha_{1}+2 \alpha_{2}\right)} \\
a_{2}= & -\frac{4 \alpha_{1} \alpha_{3} b_{0} b_{2}+\alpha_{1} \alpha_{3} b_{1}^{2}+2 \alpha_{0} \alpha_{1} b_{2}+4 \alpha_{0} \alpha_{2} b_{2}-8 \alpha_{3} \alpha_{4} b_{2}}{4 \mu^{2} b_{2} \alpha_{1}\left(\alpha_{1}+2 \alpha_{2}\right)} \\
a_{1}= & -\frac{b_{1}\left(12 \alpha_{1} \alpha_{3} b_{0} b_{2}-\alpha_{1} \alpha_{3} b_{1}^{2}+6 \alpha_{0} \alpha_{1} b_{2}+12 \alpha_{0} \alpha_{2} b_{2}-24 \alpha_{3} \alpha_{4} b_{2}\right)}{12 \mu^{2} \alpha_{1} b_{2}^{2}\left(\alpha_{1}+\alpha_{2}\right)} \\
a_{0}= & -\frac{1}{48 \mu^{3} \alpha_{1}^{2} \alpha_{2} b_{2}^{3}\left(\alpha_{1}+2 \alpha_{2}\right)\left(7 \alpha_{1}-6 \alpha_{2}\right)}\left(336 \mu \alpha_{1}^{3} \alpha_{3}^{2} b_{0}^{2} b_{2}^{2}-84 \mu \alpha_{1}^{3} \alpha_{3}^{2} b_{0} b_{1}^{2} b_{2}+\right. \\
& 7 \mu \alpha_{1}^{3} \alpha_{3}^{2} b_{1}^{4}-288 \mu \alpha_{1}^{2} \alpha_{2} \alpha_{3}^{2} b_{0}^{2} b_{2}^{2}+72 \mu \alpha_{1}^{2} \alpha_{2} \alpha_{3}^{2} b_{0} b_{1}^{2} b_{2}-6 \mu \alpha_{1}^{2} \alpha_{2} \alpha_{3}^{2} b_{1}^{4}+ \\
& 336 \mu \alpha_{0} \alpha_{1}^{3} \alpha_{3} b_{0} b_{2}^{2}-42 \mu \alpha_{0} \alpha_{1}^{3} \alpha_{3} b_{1}^{2} b_{2}+384 \mu \alpha_{0} \alpha_{1}^{2} \alpha_{2} \alpha_{3} b_{0} b_{2}^{2}-48 \mu \alpha_{0} \alpha_{1}^{2} \alpha_{2} \alpha_{3} b_{1}^{2} b_{2}- \\
& 576 \mu \alpha_{0} \alpha_{1} \alpha_{2}^{2} \alpha_{3} b_{0} b_{2}^{2}+72 \mu \alpha_{0} \alpha_{1} \alpha_{2}^{2} \alpha_{3} b_{1}^{2} b_{2}-1344 \mu \alpha_{1}^{2} \alpha_{3}^{2} \alpha_{4} b_{0} b_{2}^{2}+168 \mu \alpha_{1}^{2} \alpha_{3}^{2} \alpha_{4} b_{1}^{2} b_{2}+ \\
& 1152 \mu \alpha_{1} \alpha_{2} \alpha_{3}^{2} \alpha_{4} b_{0} b_{2}^{2}-144 \mu \alpha_{1} \alpha_{2} \alpha_{3}^{2} \alpha_{4} b_{1}^{2} b_{2}+24 \mu \alpha_{0}^{2} \alpha_{1}^{3} b_{2}^{2}+144 \mu \alpha_{0}^{2} \alpha_{1}^{2} \alpha_{2} b_{2}^{2}+ \\
& 288 \mu \alpha_{0}^{2} \alpha_{1} \alpha_{2}^{2} b_{2}^{2}+192 \mu \alpha_{0}^{2} \alpha_{2}^{3} b_{2}^{2}-672 \mu \alpha_{0} \alpha_{1}^{2} \alpha_{3} \alpha_{4} b_{2}^{2}-1728 \mu \alpha_{0} \alpha_{1} \alpha_{2} \alpha_{3} \alpha_{4} b_{2}^{2}- \\
& 768 \mu \alpha_{0} \alpha_{2}^{2} \alpha_{3} \alpha_{4} b_{2}^{2}+2304 \mu \alpha_{1} \alpha_{3}^{2} \alpha_{4}^{2} b_{2}^{2}+768 \mu \alpha_{2} \alpha_{3}^{2} \alpha_{4}^{2} b_{2}^{2}+240 \mu \alpha_{1}^{3} \alpha_{3} b_{2}^{2}+ \\
& \left.480 \mu \alpha_{1}^{2} \alpha_{2} \alpha_{3} b_{2}^{2}+240 \nu \alpha_{1}^{3} \alpha_{3} b_{2}^{2}+480 \nu \alpha_{1}^{2} \alpha_{2} \alpha_{3} b_{2}^{2}\right)
\end{aligned}
$$

and the solution of Eq.(4.18) for the discussed particular case (4.35) is

$$
u(\xi)=b_{0}+b_{1} V_{a_{0}, a_{1}, a_{2}, a_{3}, a_{4}}(\xi ; 1,2,4)+b_{2} V_{a_{0}, a_{1}, a_{2}, a_{3}, a_{4}}^{2}(\xi ; 1,2,4)
$$

Let us now consider the particular case when $V_{a_{0}, a_{1}, a_{2}, a_{3}, a_{4}}(\xi ; 1,2,4)$ is reduced to the Jacobi elliptic function $\operatorname{sn}(\xi ; k)$. In this case we have to set $a_{0}=1 ; a_{1}=0 ; a_{2}=-\left(1+k^{2}\right) ; a_{3}=0 ; a_{4}=k^{2}$ in Eqs. (A.2). The full solution of the system of algebraic equation is again very long. In order to illustrate it we discuss the particular case given by Eq.(4.35). A solution of the obtained system is

$$
\begin{aligned}
b_{0}= & \frac{1}{2 \mu \alpha_{1} \alpha_{3}\left(7 \alpha_{1}-6 \alpha_{2}\right)}\left[21 \mu^{3} \alpha_{1}^{3}+24 \mu^{3} \alpha_{1}^{2} \alpha_{2}-36 \mu^{3} \alpha_{1} \alpha_{2}^{2}-7 \mu \alpha_{0} \alpha_{1}^{2}-\right. \\
& 8 \mu \alpha_{0} \alpha_{1} \alpha_{2}+12 \mu \alpha_{0} \alpha_{2}^{2}+28 \alpha_{4} \mu \alpha_{3} \alpha_{1}-24 \mu \alpha_{2} \alpha_{3} \alpha_{4}+\left(-\left(7 \alpha_{1}^{2}+8 \alpha_{1} \alpha_{2}-12 \alpha_{2}^{2}\right) \mu \times\right. \\
& \left(21 \mu^{5} \alpha_{1}^{4}+24 \mu^{5} \alpha_{1}^{3} \alpha_{2}-36 \mu^{5} \alpha_{1}^{2} \alpha_{2}^{2}-5 \mu \alpha_{0}^{2} \alpha_{1}^{2}+20 \mu \alpha_{0}^{2} \alpha_{2}^{2}-80 \mu \alpha_{0} \alpha_{2} \alpha_{3} \alpha_{4}+80 \mu \alpha_{3}^{2} \alpha_{4}^{2}+\right. \\
& \left.\left.\left.20 \mu \alpha_{1}^{2} \alpha_{3}+20 \nu \alpha_{1}^{2} \alpha_{3}\right)\right)^{1 / 2}\right]
\end{aligned}
$$




$$
\begin{aligned}
b_{1}= & 0 \\
b_{2}= & -\frac{3}{2 \mu \alpha_{1} \alpha_{3}\left(7 \alpha_{1}-6 \alpha_{2}\right)}\left[\mu^{3} \alpha_{1}\left(7 \alpha_{1}^{2}+8 \alpha_{1} \alpha_{2}-12 \alpha_{2}^{2}\right)+\left(-\left(7 \alpha_{1}^{2}+8 \alpha_{1} \alpha_{2}-12 \alpha_{2}^{2}\right) \mu \times\right.\right. \\
& \left(21 \mu^{5} \alpha_{1}^{4}+24 \mu^{5} \alpha_{1}^{3} \alpha_{2}-36 \mu^{5} \alpha_{1}^{2} \alpha_{2}^{2}-5 \mu \alpha_{0}^{2} \alpha_{1}^{2}+20 \mu \alpha_{0}^{2} \alpha_{2}^{2}-80 \mu \alpha_{0} \alpha_{2} \alpha_{3} \alpha_{4}+80 \mu \alpha_{3}^{2} \alpha_{4}^{2}+\right. \\
& \left.\left.\left.20 \mu \alpha_{1}^{2} \alpha_{3}+20 \nu \alpha_{1}^{2} \alpha_{3}\right)\right)^{1 / 2}\right] \\
k= & \frac{1}{\sqrt{2} \mu \alpha_{1}\left(\alpha_{1}+2 \alpha_{2}\right)}\left[\frac { 1 } { \mu ( 7 \alpha _ { 1 } - 6 \alpha _ { 2 } ) } \left(\alpha _ { 1 } ( \alpha _ { 1 } + 2 \alpha _ { 2 } ) \left(7 \mu^{3} \alpha_{1}^{3}+8 \mu^{3} \alpha_{1}^{2} \alpha_{2}-12 \mu^{3} \alpha_{1} \alpha_{2}^{2}+\right.\right.\right. \\
& \left(-\left(7 \alpha_{1}^{2}+8 \alpha_{1} \alpha_{2}-12 \alpha_{2}^{2}\right) \mu\left(21 \mu^{5} \alpha_{1}^{4}+24 \mu^{5} \alpha_{1}^{3} \alpha_{2}-36 \mu^{5} \alpha_{1}^{2} \alpha_{2}^{2}-5 \mu \alpha_{0}^{2} \alpha_{1}^{2}+20 \mu \alpha_{0}^{2} \alpha_{2}^{2}-\right.\right. \\
& \left.\left.\left.\left.\left.80 \mu \alpha_{0} \alpha_{2} \alpha_{3} \alpha_{4}+80 \mu \alpha_{3}^{2} \alpha_{4}^{2}+20 \mu \alpha_{1}^{2} \alpha_{3}+20 \nu \alpha_{1}^{2} \alpha_{3}\right)\right)^{1 / 2}\right)\right)\right]^{1 / 2}
\end{aligned}
$$

and the solution of Eq.(4.18) is

$$
\begin{aligned}
u(\xi)= & \frac{1}{2 \mu \alpha_{1} \alpha_{3}\left(7 \alpha_{1}-6 \alpha_{2}\right)}\left[21 \mu^{3} \alpha_{1}^{3}+24 \mu^{3} \alpha_{1}^{2} \alpha_{2}-36 \mu^{3} \alpha_{1} \alpha_{2}^{2}-7 \mu \alpha_{0} \alpha_{1}^{2}-\right. \\
& 8 \mu \alpha_{0} \alpha_{1} \alpha_{2}+12 \mu \alpha_{0} \alpha_{2}^{2}+28 \alpha_{4} \mu \alpha_{3} \alpha_{1}-24 \mu \alpha_{2} \alpha_{3} \alpha_{4}+\left(-\left(7 \alpha_{1}^{2}+8 \alpha_{1} \alpha_{2}-12 \alpha_{2}^{2}\right) \mu \times\right. \\
& \left(21 \mu^{5} \alpha_{1}^{4}+24 \mu^{5} \alpha_{1}^{3} \alpha_{2}-36 \mu^{5} \alpha_{1}^{2} \alpha_{2}^{2}-5 \mu \alpha_{0}^{2} \alpha_{1}^{2}+20 \mu \alpha_{0}^{2} \alpha_{2}^{2}-80 \mu \alpha_{0} \alpha_{2} \alpha_{3} \alpha_{4}+80 \mu \alpha_{3}^{2} \alpha_{4}^{2}+\right. \\
& \left.\left.20 \mu \alpha_{1}^{2} \alpha_{3}+20 \nu \alpha_{1}^{2} \alpha_{3}\right)\right)^{1 / 2}-\frac{3}{2 \mu \alpha_{1} \alpha_{3}\left(7 \alpha_{1}-6 \alpha_{2}\right)}\left[\mu^{3} \alpha_{1}\left(7 \alpha_{1}^{2}+8 \alpha_{1} \alpha_{2}-12 \alpha_{2}^{2}\right)+\right. \\
& \left(-\left(7 \alpha_{1}^{2}+8 \alpha_{1} \alpha_{2}-12 \alpha_{2}^{2}\right) \mu \times\left(21 \mu^{5} \alpha_{1}^{4}+24 \mu^{5} \alpha_{1}^{3} \alpha_{2}-36 \mu^{5} \alpha_{1}^{2} \alpha_{2}^{2}-5 \mu \alpha_{0}^{2} \alpha_{1}^{2}+\right.\right. \\
& \left.\left.\left.20 \mu \alpha_{0}^{2} \alpha_{2}^{2}-80 \mu \alpha_{0} \alpha_{2} \alpha_{3} \alpha_{4}+80 \mu \alpha_{3}^{2} \alpha_{4}^{2}+20 \mu \alpha_{1}^{2} \alpha_{3}+20 \nu \alpha_{1}^{2} \alpha_{3}\right)\right)^{1 / 2}\right] \times \\
& \operatorname{sn}^{2}\left\{\xi ; \frac{1}{\sqrt{2} \mu \alpha_{1}\left(\alpha_{1}+2 \alpha_{2}\right)}\left[\frac { 1 } { \mu ( 7 \alpha _ { 1 } - 6 \alpha _ { 2 } ) } \left(\alpha _ { 1 } ( \alpha _ { 1 } + 2 \alpha _ { 2 } ) \left(7 \mu^{3} \alpha_{1}^{3}+8 \mu^{3} \alpha_{1}^{2} \alpha_{2}-\right.\right.\right.\right. \\
& 12 \mu^{3} \alpha_{1} \alpha_{2}^{2}+\left(-\left(7 \alpha_{1}^{2}+8 \alpha_{1} \alpha_{2}-12 \alpha_{2}^{2}\right) \mu\left(21 \mu^{5} \alpha_{1}^{4}+24 \mu^{5} \alpha_{1}^{3} \alpha_{2}-36 \mu^{5} \alpha_{1}^{2} \alpha_{2}^{2}-\right.\right. \\
& \left.\left.\left.\left.\left.\left.5 \mu \alpha_{0}^{2} \alpha_{1}^{2}+20 \mu \alpha_{0}^{2} \alpha_{2}^{2}-80 \mu \alpha_{0} \alpha_{2} \alpha_{3} \alpha_{4}+80 \mu \alpha_{3}^{2} \alpha_{4}^{2}+20 \mu \alpha_{1}^{2} \alpha_{3}+20 \nu \alpha_{1}^{2} \alpha_{3}\right)\right)^{1 / 2}\right)\right)\right]^{1 / 2}\right\} \\
& \xi=\mu x+\nu t .
\end{aligned}
$$

Let us now consider the particular case when the simplest equation is the Riccati equation

$$
g_{(1)}=c_{0}+c_{1} g+c_{2} g^{2}
$$

In this case

$$
a_{0}=c_{0}^{2} ; a_{1}=2 c_{0} c_{1} ; a_{2}=c_{1}^{2}+2 c_{0} c_{2} ; a_{3}=2 c_{1} c_{2} ; a_{4}=c_{2}^{2}
$$


The full solution of the system of algebraic equation is again very long. We illustrate it for the particular case given by Eq.(4.35). A solution of the system of algebraic equations is

$$
\begin{aligned}
b_{0}= & -\frac{8 \mu^{2} \alpha_{1}^{2} c_{0} c_{2}+\mu^{2} \alpha_{1}^{2} c_{1}^{2}+16 \mu^{2} \alpha_{1} \alpha_{2} c_{0} c_{2}+2 \mu^{2} \alpha_{1} \alpha_{2} c_{1}^{2}+2 \alpha_{0} \alpha_{1}+4 \alpha_{0} \alpha_{2}-8 \alpha_{3} \alpha_{4}}{4 \alpha_{1} \alpha_{3}} \\
b_{1}= & -\frac{3 \mu^{2} c_{1} c_{2}\left(\alpha_{1}+2 \alpha_{2}\right)}{\alpha_{3}} \\
b_{2}= & -\frac{3 \mu^{2} c_{2}^{2}\left(\alpha_{1}+2 \alpha_{2}\right)}{\alpha_{3}} \\
\nu= & -\frac{\mu}{80 \alpha_{1}^{2} \alpha_{3}}\left(12 \mu^{4} \alpha_{1}^{4} c_{0}^{2} c_{2}^{2}-56 \mu^{4} \alpha_{1}^{4} c_{0} c_{1}^{2} c_{2}+7 \mu^{4} \alpha_{1}^{4} c_{1}^{4}+128 \mu^{4} \alpha_{1}^{3} \alpha_{2} c_{0}^{2} c_{2}^{2}-\right. \\
& 64 \mu^{4} \alpha_{1}^{3} \alpha_{2} c_{0} c_{1}^{2} c_{2}+8 \mu^{4} \alpha_{1}^{3} \alpha_{2} c_{1}^{4}-192 \mu^{4} \alpha_{1}^{2} \alpha_{2}^{2} c_{0}^{2} c_{2}^{2}+96 \mu^{4} \alpha_{1}^{2} \alpha_{2}^{2} c_{0} c_{1}^{2} c_{2}- \\
& \left.12 \mu^{4} \alpha_{1}^{2} \alpha_{2}^{2} c_{1}^{4}-20 \alpha_{0}^{2} \alpha_{1}^{2}+80 \alpha_{0}^{2} \alpha_{2}^{2}-320 \alpha_{0} \alpha_{2} \alpha_{3} \alpha_{4}+320 \alpha_{3}^{2} \alpha_{4}^{2}+80 \alpha_{1}^{2} \alpha_{3}\right)
\end{aligned}
$$

From the solutions of the Riccati equation (4.39) here we shall discuss the solution ( $c$ is a constant of integration)

$$
g(\xi)=\frac{1}{2 c_{2}}\left\{\tanh \left[-\frac{\left(c_{1}^{2}-4 c_{0} c_{2}\right)^{1 / 2}}{2}(\xi+c)\right]-c_{1}\right\}
$$

valid when $c_{1}^{2}>4 c_{0} c_{2}$ and $\left(2 c_{2} g(\xi)+c_{1}\right)^{2}<c_{1}^{2}-4 c_{0} c_{2}$. Then the solution of Eq.(4.18) is

$$
\begin{aligned}
u(\xi)= & -\frac{8 \mu^{2} \alpha_{1}^{2} c_{0} c_{2}+\mu^{2} \alpha_{1}^{2} c_{1}^{2}+16 \mu^{2} \alpha_{1} \alpha_{2} c_{0} c_{2}+2 \mu^{2} \alpha_{1} \alpha_{2} c_{1}^{2}+2 \alpha_{0} \alpha_{1}+4 \alpha_{0} \alpha_{2}-8 \alpha_{3} \alpha_{4}}{4 \alpha_{1} \alpha_{3}} \\
& -\frac{3 \mu^{2} c_{1}\left(\alpha_{1}+2 \alpha_{2}\right)}{2 \alpha_{3}}\left\{\tanh \left[-\frac{\left(c_{1}^{2}-4 c_{0} c_{2}\right)^{1 / 2}}{2}(\xi+c)\right]-c_{1}\right\}- \\
& -\frac{3 \mu^{2}\left(\alpha_{1}+2 \alpha_{2}\right)}{4 \alpha_{3}}\left\{\tanh \left[-\frac{\left(c_{1}^{2}-4 c_{0} c_{2}\right)^{1 / 2}}{2}(\xi+c)\right]-c_{1}\right\}^{2}
\end{aligned}
$$

which is a kink.

\subsection{Case $m=5$}

In this case $q=3$. Then

$$
u(\xi)=b_{0}+b_{1} g(\xi)+b_{2} g^{2}(\xi)+b_{3} g^{3}(\xi) ; \quad \xi=\mu x+\nu t
$$

and

$$
g_{(1)}^{2}=a_{0}+a_{1} g+a_{2} g^{2}+a_{3} g^{3}+a_{4} g^{4}+a_{5} g^{5}
$$


In addition we have to solve the system of nonlinear algebraic equations for the parameters of the solution that can be obtained from the equation $W_{1}(g)=0$ from Eqs.(4.19).

The general solution of Eq. (4.46) is given by the function $V_{a_{0}, a_{1}, a_{2}, a_{3}, a_{4}, a_{5}}(\xi ; 1,2,5)$.

The system of algebraic equations obtained from Eq.(4.18) is (A.3). The full solution of this system is quite long. We shall illustrate the solution for the particular case

$$
\alpha_{2}=\alpha_{3}=1 ; \alpha_{5}=\frac{\left(\alpha_{1}+2 \alpha_{2}\right)^{2}}{40 \alpha_{3}}=\frac{\left(\alpha_{1}+2\right)^{2}}{40}
$$

The solution for this case is

$$
\begin{aligned}
a_{5} & =-\frac{4 b_{3}}{81 \mu^{2}} \\
a_{4} & =-\frac{20 b_{2}}{243 \mu^{2}} \\
a_{3} & =-\frac{4\left(b_{2}^{2}+7 b_{1} b_{3}\right)}{243 \mu^{2} b_{3}} \\
a_{2}= & -\frac{2\left(162 \alpha_{1} b_{0} b_{3}^{2}+72 \alpha_{1} b_{1} b_{2} b_{3}-10 \alpha_{1} b_{2}^{3}+243 \alpha_{0} b_{3}^{2}-324 \alpha_{4} b_{3}^{2}\right)}{2187 \mu^{2} b_{3}^{2} \alpha_{1}} \\
a_{1}= & -\frac{4}{6561 \mu^{2} \alpha_{1} b_{3}^{3}}\left(162 \alpha_{1} b_{0} b_{2} b_{3}^{2}+108 \alpha_{1} b_{1}^{2} b_{3}^{2}-63 \alpha_{1} b_{1} b_{2}^{2} b_{3}+8 \alpha_{1} b_{2}^{4}+\right. \\
a_{0}= & -\frac{2}{6561 \mu^{2} \alpha_{1} b_{3}^{4}}\left(648 \alpha_{1} b_{0} b_{1} b_{3}^{3}-162 \alpha_{1} b_{0} b_{2}^{2} b_{3}^{2}-144 \alpha_{1} b_{1}^{2} b_{2} b_{3}^{2}+68 \alpha_{1} b_{1} b_{2}^{3} b_{3}-8 \alpha_{1} b_{2}^{5}+\right. \\
& \left.972 \alpha_{0} b_{1} b_{3}^{3}-243 \alpha_{0} b_{2}^{2} b_{3}^{2}-1296 \alpha_{4} b_{1} b_{3}^{3}+324 \alpha_{4} b_{2}^{2} b_{3}^{2}\right) \\
T^{*}= & -\frac{-65610 \nu \alpha_{1} b_{3}^{4}}{T^{*}} \\
\mu & 4374 \alpha_{1}^{2} b_{0}^{2} b_{3}^{4}-2916 \alpha_{1}^{2} b_{0} b_{1} b_{2} b_{3}^{3}+648 \alpha_{1}^{2} b_{0} b_{2}^{3} b_{3}^{2}-216 \alpha_{1}^{2} b_{1}^{3} b_{3}^{3}+702 \alpha_{1}^{2} b_{1}^{2} b_{2}^{2} b_{3}^{2}- \\
& 288 \alpha_{1}^{2} b_{1} b_{2}^{4} b_{3}+32 \alpha_{1}^{2} b_{2}^{6}+13122 \alpha_{0} \alpha_{1} b_{0} b_{3}^{4}-4374 \alpha_{0} \alpha_{1} b_{1} b_{2} b_{3}^{3}+972 \alpha_{0} \alpha_{1} b_{2}^{3} b_{3}^{2}- \\
& 17496 \alpha_{1} \alpha_{4} b_{0} b_{3}^{4}+5832 \alpha_{1} \alpha_{4} b_{1} b_{2} b_{3}^{3}-1296 \alpha_{1} \alpha_{4} b_{2}^{3} b_{3}^{2}+59049 \alpha_{0}^{2} b_{3}^{4}- \\
& 288684 \alpha_{0} \alpha_{4} b_{3}^{4}+279936 \alpha_{4}^{2} b_{3}^{4}+65610 \alpha_{1} b_{3}^{4}
\end{aligned}
$$

and the solution of Eq.(4.18) (for the particular case given by Eqs.(4.47)) is

$$
\begin{aligned}
u(\xi)= & b_{0}+b_{1} V_{a_{0}, a_{1}, a_{2}, a_{3}, a_{4}, a_{5}}(\xi ; 1,2,5)+b_{2} V_{a_{0}, a_{1}, a_{2}, a_{3}, a_{4}, a_{5}}^{2}(\xi ; 1,2,5)+ \\
& b_{3} V_{a_{0}, a_{1}, a_{2}, a_{3}, a_{4}, a_{5}}^{3}(\xi ; 1,2,5)
\end{aligned}
$$


where

$$
\xi=\frac{65610 \nu \alpha_{1} b_{3}^{4}}{T^{*}} x+\nu t
$$

\subsection{Case $m=6$}

In this case $q=4$. We note that with increasing $m$ (and $q$ ) the number of equations in the nonlinear algebraic system we have to solve becomes large very fast. When the number of equations become larger that the number of parameters of the solution then the system could not have any nontrivial solution.

For the case $m=6$

$$
u(\xi)=b_{0}+b_{1} g(\xi)+b_{2} g^{2}(\xi)+b_{3} g^{3}(\xi)+b_{4} g^{4}(\xi) ; \quad \xi=\mu x+\nu t
$$

and

$$
g_{(1)}^{2}=a_{0}+a_{1} g+a_{2} g^{2}+a_{3} g^{3}+a_{4} g^{4}+a_{5} g^{5}+a_{6} g^{6}
$$

In addition we have to solve the system of nonlinear algebraic equations for the parameters of the solution that can be obtained from the equation $W_{1}(g)=0$ from Eqs.(4.19). The general solution of Eq.(4.52) is given by the function $V_{a_{0}, a_{1}, a_{2}, a_{3}, a_{4}, a_{5}, a_{6}}(\xi ; 1,2,6)$.

The system of nonlinear algebraic equations connected to Eq.(4.18) is very large. It still has a solution. In order to illustrate this solution we shall consider a particular case of Eq.(4.52) namely the equation of Abel of first kind

$$
g_{(1)}=c_{0}+c_{1} g+c_{2} g^{2}+c_{3} g^{3}
$$

The corresponding system of nonlinear algebraic equations consists of 12 equations. The solution is very long and we shall illustrate it for the particular case when $\alpha_{5}=\frac{\left(\alpha_{1}+2 \alpha_{2}\right)^{2}}{40 \alpha_{3}}$. For this case one solution of the system of 
algebraic equations is

$$
\begin{aligned}
b_{4} & =-\frac{4 \mu^{2} c_{2}^{4}\left(\alpha_{1}+2 \alpha_{2}\right)}{3 \alpha_{3} c_{1}^{2}} \\
b_{3} & =-\frac{16 \mu^{2} c_{2}^{3}\left(\alpha_{1}+2 \alpha_{2}\right.}{3 \alpha_{3} c_{1}} \\
b_{2} & =-\frac{8 \mu^{2} c_{2}^{2}\left(\alpha_{1}+2 \alpha_{2}\right)}{\alpha_{3}} \\
b_{1} & =-\frac{16 \mu^{2} c_{2}\left(3 \alpha_{1} c_{0} c_{2}+4 \alpha_{1} c_{1}^{2}+6 \alpha_{2} c_{0} c_{2}+8 \alpha_{2} c_{1}^{2}\right)}{15 \alpha_{3} c_{1}} \\
b_{0} & =-\frac{1}{30 \alpha_{1} \alpha_{3}}\left(96 \mu^{2} \alpha_{1}^{2} c_{0} c_{2}+8 \mu^{2} \alpha_{1}^{2} c_{1}^{2}+192 \mu^{2} \alpha_{1} \alpha_{2} c_{0} c_{2}+16 \mu^{2} \alpha_{1} \alpha_{2} c_{1}^{2}+\right. \\
c_{3} & =\frac{c_{1}^{3}}{27 c_{0}^{2}} \\
c_{2} & =\frac{c_{1}^{2}}{3 c_{0}} \\
\mu & =\frac{4 \nu \alpha_{1}^{2} \alpha_{3}}{\alpha_{0}^{2} \alpha_{1}^{2}-4 \alpha_{0}^{2} \alpha_{2}^{2}+16 \alpha_{0} \alpha_{2} \alpha_{3} \alpha_{4}-16 \alpha_{3}^{2} \alpha_{4}^{2}-4 \alpha_{1}^{2} \alpha_{3}}
\end{aligned}
$$

The Abel equations becomes:

$$
g_{(1)}=c_{0}+c_{1} g+\frac{c_{1}^{2}}{3 c_{0}} g^{2}+\frac{c_{1}^{3}}{27 c_{0}^{2}} g^{3}
$$

Its solution is:

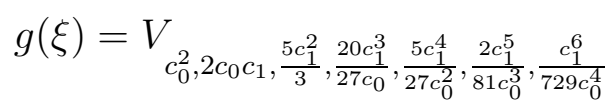

The solution of Eq. (4.18) is

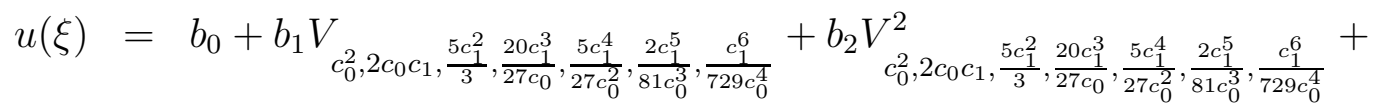

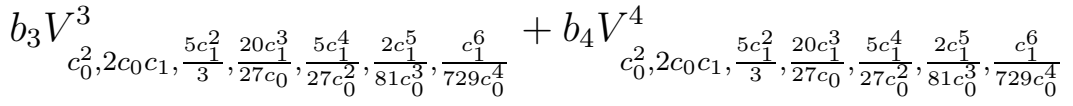

where

$$
\xi=\frac{4 \nu \alpha_{1}^{2} \alpha_{3}}{\alpha_{0}^{2} \alpha_{1}^{2}-4 \alpha_{0}^{2} \alpha_{2}^{2}+16 \alpha_{0} \alpha_{2} \alpha_{3} \alpha_{4}-16 \alpha_{3}^{2} \alpha_{4}^{2}-4 \alpha_{1}^{2} \alpha_{3}} x+\nu t
$$


Let now $c_{0}=\frac{c_{2}}{3 c_{3}}\left(c_{1}-\frac{2 c_{2}^{2}}{9 c_{3}}\right)$ and $c_{3}<0$. Then the Abel equation

$$
g_{(1)}=\frac{c_{2}}{3 c_{3}}\left(c_{1}-\frac{2 c_{2}^{2}}{9 c_{3}}+c_{1} g+c_{2} g^{2}+c_{3} g^{3}\right)
$$

has the solution [58]

$$
g(\xi)=\frac{\exp \left[\left(c_{1}-\frac{c_{2}^{2}}{3 c_{3}}\right) \xi\right]}{\sqrt{C-2 c_{3} \exp \left[2\left(c_{1}-\frac{c_{2}^{2}}{3 c_{3}}\right) \xi\right]}}-\frac{c_{2}}{3 c_{3}}
$$

where $C$ is the constant of integration. Let us assume that $C>0$. The use of the Abel equation (4.58) leads to a following solution of the system of nonlinear algebraic equations (for the particular case $\alpha_{5}=\frac{\left(\alpha_{1}+2 \alpha_{2}\right)^{2}}{40 \alpha_{3}}$ )

$$
\begin{aligned}
b_{4}= & -\frac{12 \mu^{2} c_{3}^{2}\left(\alpha_{1}+2 \alpha_{2}\right)}{\alpha_{3}} \\
b_{3}= & -\frac{16 \mu^{2} c_{2} c_{3}\left(\alpha_{1}+2 \alpha_{2}\right)}{\alpha_{3}} \\
b_{2}= & -\frac{4 \mu^{2}\left(3 \alpha_{1} c_{1} c_{3}+\alpha_{1} c_{2}^{2}+6 \alpha_{2} c_{1} c_{3}+2 \alpha_{2} c_{2}^{2}\right)}{\alpha_{3}} \\
b_{1}= & -\frac{8 \mu^{2}\left(9 \alpha_{1} c_{1} c_{3}-\alpha_{1} c_{2}^{2}+18 \alpha_{2} c_{1} c_{3}-2 \alpha_{2} c_{2}^{2}\right)}{9 \alpha_{3} c_{3}} \\
\nu= & -\frac{\mu}{1620 \alpha_{1}^{2} \alpha_{3} c_{3}^{4}}\left[2268 \mu^{4} \alpha_{1}^{4} c_{1}^{4} c_{3}^{4}-3024 \mu^{4} \alpha_{1}^{4} c_{1}^{3} c_{2}^{2} c_{3}^{3}+1512 \mu^{4} \alpha_{1}^{4} c_{1}^{2} c_{2}^{4} c_{3}^{2}-\right. \\
& 336 \mu^{4} \alpha_{1}^{4} c_{1} c_{2}^{6} c_{3}+28 \mu^{4} \alpha_{1}^{4} c_{2}^{8}+2592 \mu^{4} \alpha_{1}^{3} \alpha_{2} c_{1}^{4} c_{3}^{4}-3456 \mu^{4} \alpha_{1}^{3} \alpha_{2} c_{1}^{3} c_{2}^{2} c_{3}^{3}+ \\
& 1728 \mu^{4} \alpha[1]^{3} \alpha_{2} c_{1}^{2} c_{2}^{4} c_{3}^{2}-384 \mu^{4} \alpha_{1}^{3} \alpha_{2} c_{1} c_{2}^{6} c_{3}+32 \mu^{4} \alpha_{1}^{3} \alpha_{2} c_{2}^{8}-3888 \mu^{4} \alpha_{1}^{2} \alpha_{2}^{2} c_{1}^{4} c_{3}^{4}+ \\
& 5184 \mu^{4} \alpha_{1}^{2} \alpha_{2}^{2} c_{1}^{3} c_{2}^{2} c_{3}^{3}-2592 \mu^{4} \alpha_{1}^{2} \alpha_{2}^{2} c_{1}^{2} c_{2}^{4} c_{3}^{2}+576 \mu^{4} \alpha_{1}^{2} \alpha_{2}^{2} c_{1} c_{2}^{6} c_{3}-48 \mu^{4} \alpha_{1}^{2} \alpha_{2}^{2} c_{2}^{8}- \\
& \left.405 \alpha_{0}^{2} \alpha_{1}^{2} c_{3}^{4}+1620 \alpha_{0}^{2} \alpha_{2}^{2} c_{3}^{4}-6480 \alpha_{0} \alpha_{2} \alpha_{3} \alpha_{4} c_{3}^{4}+6480 \alpha_{3}^{2} \alpha_{4}^{2} c_{3}^{4}+1620 \alpha_{1}^{2} \alpha_{3} c_{3}^{4}\right]
\end{aligned}
$$


Hence the solution of Eq.(4.18) becomes

$$
\begin{aligned}
& u(\xi)=b_{0}-\frac{8 \mu^{2}\left(9 \alpha_{1} c_{1} c_{3}-\alpha_{1} c_{2}^{2}+18 \alpha_{2} c_{1} c_{3}-2 \alpha_{2} c_{2}^{2}\right)}{9 \alpha_{3} c_{3}} \times \\
& \left(\frac{\exp \left[\left(c_{1}-\frac{c_{2}^{2}}{3 c_{3}}\right) \xi\right]}{\sqrt{C-2 c_{3} \exp \left[2\left(c_{1}-\frac{c_{2}^{2}}{3 c_{3}}\right) \xi\right]}}-\frac{c_{2}}{3 c_{3}}\right)- \\
& -\frac{4 \mu^{2}\left(3 \alpha_{1} c_{1} c_{3}+\alpha_{1} c_{2}^{2}+6 \alpha_{2} c_{1} c_{3}+2 \alpha_{2} c_{2}^{2}\right)}{\alpha_{3}} \times \\
& \left(\frac{\exp \left[\left(c_{1}-\frac{c_{2}^{2}}{3 c_{3}}\right) \xi\right]}{\sqrt{C-2 c_{3} \exp \left[2\left(c_{1}-\frac{c_{2}^{2}}{3 c_{3}}\right) \xi\right]}}-\frac{c_{2}}{3 c_{3}}\right)^{2}- \\
& -\frac{16 \mu^{2} c_{2} c_{3}\left(\alpha_{1}+2 \alpha_{2}\right)}{\alpha_{3}}\left(\frac{\exp \left[\left(c_{1}-\frac{c_{2}^{2}}{3 c_{3}}\right) \xi\right]}{\sqrt{C-2 c_{3} \exp \left[2\left(c_{1}-\frac{c_{2}^{2}}{3 c_{3}}\right) \xi\right]}}-\frac{c_{2}}{3 c_{3}}\right)^{3}- \\
& -\frac{12 \mu^{2} c_{3}^{2}\left(\alpha_{1}+2 \alpha_{2}\right)}{\alpha_{3}}\left(\frac{\exp \left[\left(c_{1}-\frac{c_{2}^{2}}{3 c_{3}}\right) \xi\right]}{\sqrt{C-2 c_{3} \exp \left[2\left(c_{1}-\frac{c_{2}^{2}}{3 c_{3}}\right) \xi\right]}}-\frac{c_{2}}{3 c_{3}}\right)^{4}
\end{aligned}
$$

that is a solution of kink's kind as the solution (4.44).

\section{Concluding remarks}

In this article we have discussed a version of the method of simplest equation applicable to a class of nonlinear partial differential equations that are much used as model equations in the area of natural sciences. Eq.(2.9) was used as simplest equation and we have described a methodology based on the concept for balance equations. This methodology reduces the studied nonlinear partial differential equations to systems of nonlinear algebraic equations. Any nontrivial solution of the obtained system of algebraic equations leads to an exact solution of the corresponding nonlinear partial differential equation. Discussed examples have shown the effectivity of the methodology of this variant of the method of simplest equation. 


\section{A Systems of algebraic equations connected to Eq.(4.18)}

A.1 Case $m=3$

$$
\begin{aligned}
0= & \frac{3}{2} \alpha_{1} \mu^{2} a_{3} b_{1}+3 \alpha_{2} \mu^{2} b_{1} a_{3}+\alpha_{3} b_{1}^{2}+\frac{45}{2} \alpha_{5} \mu^{4} a_{3}^{2} \\
0= & 15 \mu^{4} a_{2} a_{3} \alpha_{5}+\mu^{2} a_{2} \alpha_{1} b_{1}+\mu^{2} a_{2} \alpha_{2} b_{1}+3 \mu^{2} a_{3} \alpha_{2} b_{0}+3 \mu^{2} a_{3} \alpha_{4}+ \\
& 2 \alpha_{3} b_{0} b_{1}+\alpha_{0} b_{1} \\
0= & \nu+\mu+\alpha_{0} \mu b_{0}+\frac{1}{2} \alpha_{1} \mu^{3} a_{1} b_{1}+\alpha_{2} \mu^{3} b_{0} a_{2}+\alpha_{3} \mu b_{0}^{2}+ \\
& \alpha_{4} \mu^{3} a_{2}+\alpha_{5} \mu^{5}\left(\frac{9}{2} a_{3} a_{1}+a_{2}^{2}\right)
\end{aligned}
$$

\section{A.2 Case $m=4$}

$$
\begin{aligned}
& 0=360 \mu^{4} a_{4}^{2} \alpha_{5}+6 \mu^{2} a_{4} \alpha_{1} b_{2}+12 \mu^{2} a_{4} \alpha_{2} b_{2}+\alpha_{3} b_{2}^{2} \\
& 0=2 \alpha_{1} \mu^{2} b_{2}\left(5 a_{3} b_{2}+2 a_{4} b_{1}\right)+6 \alpha_{1} \mu^{2} a_{4} b_{2} b_{1}+24 \alpha_{2} \mu^{2} b_{1} a_{4} b_{2}+ \\
& \alpha_{2} \mu^{2} b_{2}\left(15 a_{3} b_{2}+6 a_{4} b_{1}\right)+5 \alpha_{3} b_{1} b_{2}^{2}+\alpha_{5} \mu^{4}\left(840 a_{3} a_{4} b_{2}+120 a_{4}^{2} b_{1}\right) \\
& 0=2 \alpha_{0} b_{2}^{2}+2 \alpha_{1} \mu^{2}\left(4 a_{2} b_{2}+(3 / 2) a_{3} b_{1}\right) b_{2}+\alpha_{1} \mu^{2}\left(5 a_{3} b_{2}+2 a_{4} b_{1}\right) b_{1}+ \\
& 24 \alpha_{2} \mu^{2} b_{0} a_{4} b_{2}+\alpha_{2} \mu^{2} b_{1}\left(15 a_{3} b_{2}+6 a_{4} b_{1}\right)+\alpha_{2} \mu^{2} b_{2}\left(8 a_{2} b_{2}+3 a_{3} b_{1}\right)+ \\
& 2 \alpha_{3}\left(2 b_{0} b_{2}+b_{1}^{2}\right) b_{2}+2 \alpha_{3} b_{1}^{2} b_{2}+24 \alpha_{4} \mu^{2} a_{4} b_{2}+\alpha_{5} \mu^{4}\left(480 a_{2} a_{4} b_{2}+210 a_{3}^{2} b_{2}+\right. \\
& \left.120 a_{3} a_{4} b_{1}\right) \\
& 0=3 \alpha_{0} b_{1} b_{2}+2 \alpha_{1} \mu^{2}\left(3 a_{1} b_{2}+a_{2} b_{1}\right) b_{2}+\alpha_{1} \mu^{2}\left(4 a_{2} b_{2}+(3 / 2) a_{3} b_{1}\right) b_{1}+ \\
& \alpha_{2} \mu^{2} b_{0}\left(15 a_{3} b_{2}+6 a_{4} b_{1}\right)+\alpha_{2} \mu^{2} b_{1}\left(8 a_{2} b_{2}+3 a_{3} b_{1}\right)+\alpha_{2} \mu^{2} b_{2}\left(3 a_{1} b_{2}+a_{2} b_{1}\right)+ \\
& 4 \alpha_{3} b_{0} b_{1} b_{2}+\alpha_{3} b_{1}\left(2 b_{0} b_{2}+b_{1}^{2}\right)+\alpha_{4} \mu^{2}\left(15 a_{3} b_{2}+6 a_{4} b_{1}\right)+\alpha_{5} \mu^{4}\left[(45 / 2) a_{3}^{2} b_{1}+\right. \\
& \left.270 a_{4} b_{2} a_{1}+195 a_{3} b_{2} a_{2}+60 a_{4} b_{1} a_{2}\right] \\
& 0=2(\nu+\mu) b_{2}+2 \alpha_{0} \mu b_{0} b_{2}+\alpha_{0} \mu b_{1}^{2}+2 \alpha_{1} \mu^{3}\left(2 a_{0} b_{2}+(1 / 2) a_{1} b_{1}\right) b_{2}+ \\
& \alpha_{1} \mu^{3}\left(3 a_{1} b_{2}+a_{2} b_{1}\right) b_{1}+\alpha_{2} \mu^{3} b_{0}\left(8 a_{2} b_{2}+3 a_{3} b_{1}\right)+\alpha_{2} \mu^{3} b_{1}\left(3 a_{1} b_{2}+a_{2} b_{1}\right)+ \\
& 2 \alpha_{3} \mu b_{0}^{2} b_{2}+2 \alpha_{3} \mu b_{0} b_{1}^{2}+\alpha_{4} \mu^{3}\left(8 a_{2} b_{2}+3 a_{3} b_{1}\right)+\alpha_{5} \mu^{5}\left(144 a_{0} a_{4} b_{2}+\right. \\
& \left.84 a_{1} a_{3} b_{2}+30 a_{1} a_{4} b_{1}+32 a_{2}^{2} b_{2}+15 a_{2} a_{3} b_{1}\right) \\
& 0=(\nu+\mu) b_{1}+\alpha_{0} \mu b_{0} b_{1}+\alpha_{1} \mu^{3}\left(2 a_{0} b_{2}+(1 / 2) a_{1} b_{1}\right) b_{1}+\alpha_{2} \mu^{3} b_{0}\left(3 a_{1} b_{2}+a_{2} b_{1}\right)+ \\
& \alpha_{3} \mu b_{0}^{2} b_{1}+\alpha_{4} \mu^{3}\left(3 a_{1} b_{2}+a_{2} b_{1}\right)+\alpha_{5} \mu^{5}\left(a_{2}^{2} b_{1}+12 a_{4} b_{1} a_{0}+15 a_{2} b_{2} a_{1}+\right. \\
& \left.30 a_{3} b_{2} a_{0}+(9 / 2) a_{3} b_{1} a_{1}\right)
\end{aligned}
$$




\section{A.3 Case $m=5$}

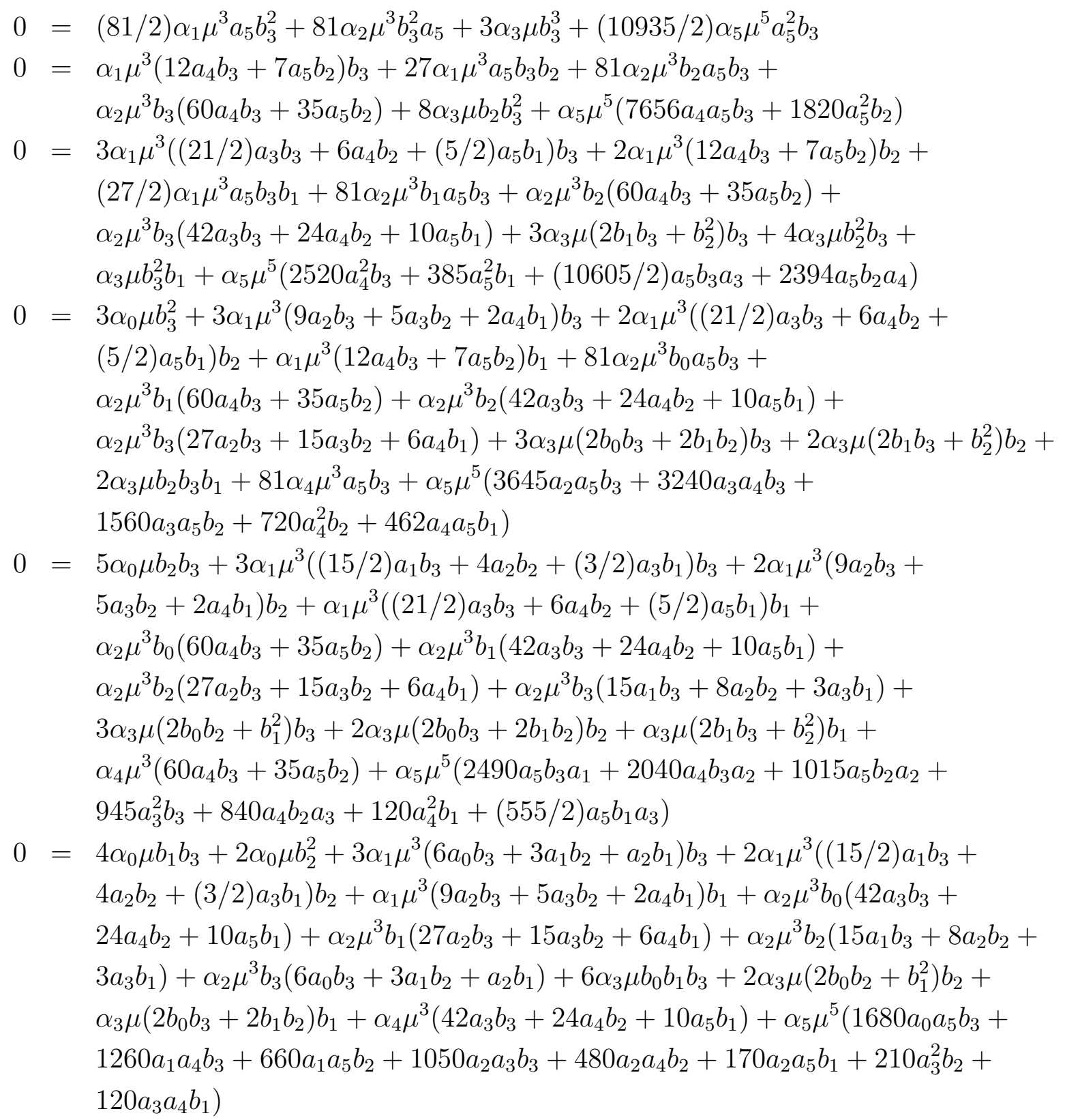




$$
\begin{aligned}
& =(3(\nu+\mu)) b_{3}+3 \alpha_{0} \mu b_{0} b_{3}+3 \alpha_{0} \mu b_{1} b_{2}+3 \alpha_{1} \mu^{3}\left(2 a_{0} b_{2}+(1 / 2) a_{1} b_{1}\right) b_{3}+ \\
& 2 \alpha_{1} \mu^{3}\left(6 a_{0} b_{3}+3 a_{1} b_{2}+a_{2} b_{1}\right) b_{2}+\alpha_{1} \mu^{3}\left((15 / 2) a_{1} b_{3}+4 a_{2} b_{2}+\right. \\
& \left.(3 / 2) a_{3} b_{1}\right) b_{1}+\alpha_{2} \mu^{3} b_{0}\left(27 a_{2} b_{3}+15 a_{3} b_{2}+6 a_{4} b_{1}\right)+\alpha_{2} \mu^{3} b_{1}\left(15 a_{1} b_{3}+8 a_{2} b_{2}+3 a_{3} b_{1}\right)+ \\
& \alpha_{2} \mu^{3} b_{2}\left(6 a_{0} b_{3}+3 a_{1} b_{2}+a_{2} b_{1}\right)+3 \alpha_{3} \mu b_{0}^{2} b_{3}+4 \alpha_{3} \mu b_{0} b_{1} b_{2}+\alpha_{3} \mu\left(2 b_{0} b_{2}+b_{1}^{2}\right) b_{1}+ \\
& \alpha_{4} \mu^{3}\left(27 a_{2} b_{3}+15 a_{3} b_{2}+6 a_{4} b_{1}\right)+\alpha_{5} \mu^{5}\left(243 a_{2}^{2} b_{3}+(45 / 2) a_{3}^{2} b_{1}+756 a_{4} b_{3} a_{0}+\right. \\
& \left.420 a_{5} b_{2} a_{0}+(1107 / 2) a_{3} b_{3} a_{1}+270 a_{4} b_{2} a_{1}+105 a_{5} b_{1} a_{1}+195 a_{3} b_{2} a_{2}+60 a_{4} b_{1} a_{2}\right) \\
& 0=(2(\nu+\mu)) b_{2}+2 \alpha_{0} \mu b_{0} b_{2}+\alpha_{0} \mu b_{1}^{2}+2 \alpha_{1} \mu^{3}\left(2 a_{0} b_{2}+(1 / 2) a_{1} b_{1}\right) b_{2}+ \\
& \alpha_{1} \mu^{3}\left(6 a_{0} b_{3}+3 a_{1} b_{2}+a_{2} b_{1}\right) b_{1}+\alpha_{2} \mu^{3} b_{0}\left(15 a_{1} b_{3}+8 a_{2} b_{2}+3 a_{3} b_{1}\right)+ \\
& \alpha_{2} \mu^{3} b_{1}\left(6 a_{0} b_{3}+3 a_{1} b_{2}+a_{2} b_{1}\right)+2 \alpha_{3} \mu b_{0}^{2} b_{2}+2 \alpha_{3} \mu b_{0} b_{1}^{2}+\alpha_{4} \mu^{3}\left(15 a_{1} b_{3}+8 a_{2} b_{2}+3 a_{3} b_{1}\right)+ \\
& \alpha_{5} \mu^{5}\left(270 a_{0} a_{3} b_{3}+144 a_{0} a_{4} b_{2}+60 a_{0} a_{5} b_{1}+195 a_{1} a_{2} b_{3}+84 a_{1} a_{3} b_{2}+30 a_{1} a_{4} b_{1}+\right. \\
& \left.32 a_{2}^{2} b_{2}+15 a_{2} a_{3} b_{1}\right) \\
& 0=(\nu+\mu) b_{1}+\alpha_{0} \mu b_{0} b_{1}+\alpha_{1} \mu^{3}\left(2 a_{0} b_{2}+(1 / 2) a_{1} b_{1}\right) b_{1}+\alpha_{2} \mu^{3} b_{0}\left(6 a_{0} b_{3}+3 a_{1} b_{2}+a_{2} b_{1}\right)+ \\
& \alpha_{3} \mu b_{0}^{2} b_{1}+\alpha_{4} \mu^{3}\left(6 a_{0} b_{3}+3 a_{1} b_{2}+a_{2} b_{1}\right)+\alpha_{5} \mu^{5}\left((45 / 2) a_{1}^{2} b_{3}+a_{2}^{2} b_{1}+(9 / 2) a_{3} b_{1} a_{1}+\right. \\
& \left.15 a_{2} b_{2} a_{1}+12 a_{4} b_{1} a_{0}+60 a_{2} b_{3} a_{0}+30 a_{3} b_{2} a_{0}\right)
\end{aligned}
$$

\section{References}

[1] A. Medio, M. Lines. Nonlinear dynamics. A primer. Cambridge University Press, Cambrifge, 2001

[2] R. Ball, N. Akhmediev. Nonlinear dynamics: From lasers to butterflies. World Scientific, Singapore, 2003

[3] H. Kantz, D Holstein, M Ragwitz, N.K. Vitanov. Markov chain model for turbulent wind speed data. Physica A 342 (2004) 315-321.

[4] Z.I. Dimitrova, N.K. Vitanov. Influence of adaptation on the nonlinear dynamics of a system of competing populations. Physics Letters A 272 (2000) 368-380.

[5] Z.I. Dimitrova, N.K. Vitanov. Adaptation and its impact on the dynamics of a system of three competing populations. Physica A 300 (2001), 91-115 .

[6] Z.I. Dimitrova, N.K. Vitanov. Chaotic pairwise competition Theoretical Population Biology 66 (2004) 1-12.

[7] N.K. Vitanov, Z.I. Dimitrova, H Kantz. On the trap of extinction and its elimination. Physics Letters A 349 (2006) 350-355. 
[8] Z.I. Dimitrova, N.K. Vitanov.Dynamical consequences of adaptation of the growth rates in a system of three competing populations. Journal of Physics A: Mathematical and General 34 (2001), 7459.

[9] N. K. Vitanov, F.H. Busse. Bounds on the heat transport in a horizontal fluid layer with stress-free boundaries. Zeitschrift fü $\mathrm{r}$ Angewandte Mathematik und Physik, ZAMP, 48 (1997), 310-324.

[10] N. K. Vitanov. Breather and soliton wave families for the sine - Gordon equation. Proceedings of the Royal Society of London A , 454 (1998), $2409-2423$.

[11] N.K. Vitanov, Z.I. Dimitrova, M. Ausloos. Verhulst - Lotka - Volterra (VLV) model of ideological struggle. Physica A 389 (2010), 4970-4980.

[12] N.K. Vitanov. Upper bounds on convective heat transport in a rotating fluid layer of infinite Prandtl number: Case of intermediate Taylor numbers Physical Review E 62 (2000), 3581 - 3591

[13] N.K. Vitanov, M. Ausloos, G. Rotundo. Discrete model of ideological struggle accounting for migration. Advances in Complex Systems 15 (2012), 1250049.

[14] J. D. Murray. Lectures on Nonlinear Differential Equation Models in Biology. Oxford University Press, Oxford, 1977.

[15] N. Martinov, N. Vitanov. On the correspondence between the selfconsistent 2D Poisson-Boltzmann structures and the sine-Gordon waves. Journal of Physics A: Mathematical and General 25 (1992), L51.

[16] N. Martinov, N. Vitanov. On some solutions of the two-dimensional sineGordon equation. Journal of Physics A: Mathematical and General 25 (1992), L419

[17] N. Martinov, N. Vitanov. Running wave solutions of the two-dimensional sine-Gordon equation. Journal of Physics A: Mathematical and General 25 (1992), 3609

[18] E. Infeld, G. Rowlands. Nonlinear Waves, Solitons and Chaos. Cambridge University Press, Cambridge, 1990.

[19] M. Ablowitz, P. A. Clarkson. Solitons, Nonlinear Evolution Equations and Inverse Scattering. Cambridge University Press, Cambridge, 1991. 
[20] N. K. Vitanov, I. P. Jordanov, Z. I. Dimitrova. On nonlinear population waves. Applied Mathematics and Computation. 215 (2009) 2950 - 2964.

[21] N. A. Kudryashov. Exact solutions of the generalized Kuramoto Sivashinsky equation. Phys. Lett. A 147 (1990) 287 - 291.

[22] A. C. Scott. Neuroscience: A Mathematical Primer. Springer, New York,2002.

[23] M. Tabor. Chaos and Integrability in Dynamical Systems. Wiley, New York, 1989.

[24] N. K. Vitanov, I. P. Jordanov, Z. I. Dimitrova. On nonlinear dynamics of interacting populations: Coupled kink waves in a system of two populations. Commun. Nonlinear Sci. Numer. Simulat. 14 (2009) 2379 $-2388$.

[25] M. J. Ablowitz, D. J. Kaup, A. C. Newell. Nonlinear evolution equations of physical significance. Phys. Rev. Lett. 31 (1973) 125 - 127.

[26] C. S. Gardner, J. M. Greene, M. D. Kruskal, R. R. Miura. Method for solving Korteweg-de Vries equation. Phys. Rev. Lett. 19 (1967) 1095 1097.

[27] M. J. Ablowitz, D. J. Kaup, A. C. Newell, H. Segur. Inverse scattering transform - Fourier analysis for nonlinear problems. Studies in Applied Mathematics 53 (1974) 249 - 315.

[28] M. Remoissenet. Waves Called Solitons. Springer, Berlin, 1993.

[29] R. Hirota. Exact solution of Korteweg-de Vries equation for multiple collisions of solitons. Phys. Rev. Lett. 27 (1971) $1192-1194$.

[30] A. M. Wazwaz. Integrability: Mathematical methods for studying solitary waves theory. Physica Scripta 89, Art. No. 038001 (2014).

[31] N. A. Kudryashov. Simplest equation method to look for exact solutions of nonlinear differential equations. Chaos Solitons \& Fractals 24 (2005) $1217-1231$.

[32] N. A. Kudryashov, N. B. Loguinova. Extended simplest equation method for nonlinear differential equations. Applied Mathematics and Computation 205 (2008) $396-402$. 
[33] N. A. Kudryashov, M. V. Demina. Polygons of differential equations for finding exact solutions. Chaos Solitons \& Fractals 33 (2007) $480-496$.

[34] N. A. Kudryashov. Meromorphic solutions of nonlinear ordinary differential equations. Communications in Nonlinear Science and Numerical Simulation 15 (2010) 2778 - 2790.

[35] N. K. Vitanov, Z. I. Dimitrova, H. Kantz. Modified method of simplest equation and its application to nonlinear PDEs. Applied Mathematics and Computation 216 (2010) $2587-2595$.

[36] N. K. Vitanov. Modified method of simplest equation: Powerful tool for obtaining exact and approximate traveling-wave solutions of nonlinear PDEs. Commun. Nonlinear Sci. Numer. Simulat. 16 (2011) 1176 - 1185.

[37] N. K. Vitanov. On modified method of simplest equation for obtaining exact and approximate solutions of nonlinear PDEs: The role of the simplest equation. Commun. Nonlinear Sci. Numer. Simulat. 16 (2011) $4215-4231$.

[38] N. A. Kudryashov. Painleve analysis and exact solutions of the Korteweg-de Vries equation with a source. Applied Mathematics Letters 41 (2015) $41-45$.

[39] N. K. Vitanov. Application of simplest equations of Bernoulli and Riccati kind for obtaining exact traveling wave solutions for a class of PDEs with polynomial nonlinearity. Commun. Nonlinear Sci. Numer. Simulat. 15 (2010) $2050-2060$.

[40] N. K. Vitanov, Z. I. Dimitrova. Application of the method of simplest equation for obtaining exact traveling-wave solutions for two classes of model PDEs from ecology and population dynamics. Commun. Nonlinear Sci. Numer. Simulat. 15 (2010) 2836 - 2845.

[41] A. Kochendörfer, A. Seeger. Theorie der Versetzungen in eindimensionalen Atomreihen. I. Periodisch angeordnete Versetzungen. (Theory of dislocations in one-dimensional atom chains. I. Periodic dislocations.) Z. Phys. 127 (1950) $533-550$.

[42] G. L. lamb, Jr. Elements of Soliton Theory. Wiley, New York, 1980.

[43] N. K. Martinov, N. K. Vitanov. New class of running wave solutions of (2+1)-dimensional sine-Gordon equation. J. Phys. A: Math. Gen. 27 (1994), $4611-4618$. 
[44] N. K. Vitanov. On travelling waves and double-periodic structures in two-dimensional sine-Gordon systems. J. Phys. A: Math. Gen. 29 (1996), $5195-5207$.

[45] N. A. Kudryashov. Exact solitary waves of the Fisher equation. Phys. Lett. A 342 (2005) 99 - 106.

[46] N. K. Vitanov, Z. I. Dimitrova, K. N. Vitanov. On the class of nonlinear PDEs that can be treated by the modified method of simplest equation. Application to generalized Degasperis - Processi equation and b-equation. Commun. Nonlinear Sci. Numer. Simulat. 16 (2011) 3033 3044 .

[47] N. K. Vitanov, Z. I. Dimitrova, H. Kantz. Application of the method of simplest equation for obtaining exact traveling-wave solutions for the extended Korteweg-de Vries equation and generalized Camassa-Holm equation. Applied Mathematics and Computation 219 (2013) 7480 7492 .

[48] N. K. Vitanov, Z. I. Dimitrova, K. N. Vitanov. Traveling waves and statistical distributions connected to systems of interacting populations. Computers \& Mathematics with Applications 66 (2013) 1666 - 1684.

[49] N. A. Kudryashov. One method for finding exact solutions of nonlinear differential equations. Commun. Nonl. Sci. Numer. Simulat. 17 (2012) $2248-2253$.

[50] N. A. Kudryashov, D. I. Sinelshchikov. Nonlinear differential equations of the second, third and fourth order with exact solutions. Applied Mathematics and Computation 218 (2012) 10454 - 10467

[51] N. K. Vitanov. Solitary wave solutions for nonlinear partial differential equations that contain monomials of odd and even grades with respect to participating derivatives. Applied Mathematics and Computation 247 (2014) $213-217$.

[52] C. F. Faa di Bruno. Note sur une nouvelle formule du caclul differentiel. Quart. J. Pure Appl. Math. 1 (1857), 359 - 360.

[53] T. L. Perelman, A. Kh. Fridman, M. M. Elyashevich. A modified Korteweg-deVries equation in electrohydrodynamics. Soviet Physics JETP (1974) 39, No. 4, 643 - 646. 
[54] P. J. Olver. Hamiltonian and non-Hamiltonian models for water waves. p.p. 273 -290 in P. G. Ciarlet, M. Roseau (eds.) Trends and applications of pure mathematics to mechanics. Lecture Notes in Physics 195, Springer, Berlin , 1984.

[55] N. A. Kudryashov, D. I. Sinelshchikov. Extended models of non-linear waves in liquid with gas bubbles. International Journal of Non-Linear Mechanics, 63 (2014) 31-38.

[56] N. A. Kudryashov, D. I. Sinelshchikov. Special solutions of a high-order equation for waves in a liquid with gas bubbles. Regular and Chaotic Dynamics, 19, No. 5 2014, 576-585.

[57] N. A. Kudryashov, M. B. Soukharev, M. V. Demina. Elliptic traveling waves of the Olver equation. Commun. Nonlinear Sci. Numer. Simulat. 17 (2012) $4104-4114$.

[58] E. Kamke. Differentialgleichungen. Lösungsmethoden und Lösungen. Bd. 1 Gewönliche Differentialgleichungen. Teubner, Berlin, 1983. 